\title{
Quantum Feedback in a Weakly Driven Cavity QED System
}

\author{
J. E. Reiner, ${ }^{1}$ W. P. Smith, ${ }^{1}$ L. A. Orozco, ${ }^{2,1}$ H. M. Wiseman,,${ }^{3,1}$ and Jay Gambetta ${ }^{3}$ \\ ${ }^{1}$ Department of Physics and Astronomy, State University of New York, Stony Brook, NY 11794-3800, USA \\ 2 Department of Physics, University of Maryland, College Park, MD 20742, USA \\ ${ }^{3}$ Centre for Quantum Computer Technology, Centre for Quantum Dynamics, \\ School of Science, Griffith University, Brisbane 4111, Australia
}

(Dated: May 9, 2004)

\begin{abstract}
Quantum feedback in strongly coupled systems can probe a regime where one quantum of excitation is a large fluctuation. We present theoretical and experimental studies of quantum feedback in an optical cavity QED system. The time evolution of the conditional state, following a photodetection, can be modified by changing the drive of the cavity. For the appropriate feedback, the conditional state can be captured in a new steady state and then released. The feedback protocol requires resonance operation, proper amplitude and delay for the change in the drive. We demonstrate the successful use of feedback in the suppression of the vacuum Rabi oscillations for the length of the feedback pulse and their subsequent return to steady state. The feedback works only because we have an entangled quantum system, rather than an analogous correlated classical system.
\end{abstract}

PACS numbers: 42.50.Lc, 42.50.Dv,03.65.Ta,03.67.-a

\section{INTRODUCTION}

Quantum feedback describes the application of a control signal related to the fluctuations of the quantum degrees of freedom of a system. For linear systems, or systems which can be linearized (because the fluctuations in the relevant quantities are small compared to their mean values), the feedback can be understood in an essentially classical way. Quantum physics enters only to dictate the magnitude of the fluctuations. Such systems have been much studied experimentally and theoretically in quantum optics since the pioneering work of Refs. [1-3]. However, nonlinear quantum systems with large fluctuations require a quite different approach to quantum feedback $[4,5]$. Cavity QED in the strong coupling limit [6] has nonlinear dynamics, and if it is weakly driven then the mean excitation number is much less than one. In this case a fluctuation in the excitation induced by the detection of one photon is large compared to the steady-state mean excitation $[7,8]$. To implement feedback in such a system, as was done recently by our group in Smith et al. [9], it is necessary to design the protocol using the conditional quantum state; that is, the non-equilibrium quantum state prepared by the detection of a photon at a random time according to the theory of quantum trajectories [10].

The simplest way to apply quantum trajectories to the theory of feedback is to derive a feedback-modified master equation $[4,5]$ that applies in the limit of Markovian feedback; that is, instantaneously feeding back the measured current. This theory has been applied for a variety of purposes. Several groups [11-16] have proposed methods for protecting quantum-optical states from decoherence. Others [17-19] developed feedback schemes to manipulate the position of an atom or mirror in a light field. Thomsen and Wiseman [20] proposed a feedback scheme that creates deterministic spin squeezing, while Reiner et al. [21] explored a protocol that modified the fluorescence spectrum of a single-atom, strongly coupled, strongly driven cavity QED system.

A more sophisticated way to apply quantum trajectory theory to quantum feedback is to use the conditioned quantum state as the basis for determining the feedback. The state is a function of the entire measurement record, so this sort of feedback is non-Markovian. This idea lay behind the proposal by Wiseman to measure phase using adaptive dyne detection [22]. Doherty et al. [23, 24] showed that basing the feedback on the conditioned state was the optimal way to control quantum systems. This work, and that of Ref. [25], showed that state-based feedback is often superior to Markovian feedback.

It is state-based feedback that has proven to be the most useful for recent experimental advances in quantum feedback. Armen et al. [26] realized the adaptive phase measurement scheme of Wiseman [22]. At the same time, we demonstrated that feedback in a strongly coupled and weakly driven cavity QED system could stabilize the nonequilibrium state conditioned on the detection of a single photon. This is the first demonstration of the control of a "deep" quantum system by feedback of a measurement result. That is, there is no equivalent classical model; the feedback can only be understood from the quantum trajectory of the system [9].

Quantum trajectories have previously been applied in this system to calculate and understand the correlation functions [27] which have also been measured experimentally $[28,29]$. The link between correlation functions and conditioned states is as follows. Consider the $g^{(2)}(\tau)$ correlation function [30]. This is an autocorrelation function for the photocurrent

$$
g^{(2)}(\tau)=\frac{\langle I(t+\tau) I(t)\rangle_{\mathrm{ss}}}{\langle I(t)\rangle_{\mathrm{ss}}^{2}}
$$

However, because $I(t)$ is zero except at those times when a photon is detected, we can rewrite this in terms of 
conditional measurements as

$$
g^{(2)}(\tau)=\frac{\langle I(t+\tau)\rangle_{\mathrm{c}}}{\langle I\rangle_{\mathrm{ss}}}
$$

where here the subscript $\mathrm{c}$ means "given a detection at time $t$ in steady state". That is, it is the probability for getting a second detection a time $\tau$ after the first detection, scaled by the steady-state detection probability. [From Eq. (1.1), the function for $\tau<0$ can be found by symmetry.]

As we will discuss in Sec. II, in the weakly driven cavity QED experiment of Refs. [9, 28, 29], the steady state system state is almost a pure state $\left|\psi_{\mathrm{ss}}\right\rangle$. Immediately following the first detection, the conditional state is $\left|\psi_{\mathrm{c}}(0)\right\rangle \propto \hat{a}\left|\psi_{\mathrm{ss}}\right\rangle$, where $\hat{a}$ is the annihilation operator for the cavity mode. The correlation function (1.2) can be reformulated as

$$
g^{(2)}(\tau)=\frac{\left\langle\psi_{\mathrm{c}}(\tau)\left|\hat{a}^{\dagger} \hat{a}\right| \psi_{\mathrm{c}}(\tau)\right\rangle}{\left\langle\psi_{\mathrm{ss}}\left|\hat{a}^{\dagger} \hat{a}\right| \psi_{\mathrm{ss}}\right\rangle}
$$

Here $\left|\psi_{\mathrm{c}}(\tau)\right\rangle$ is the conditional state for $\tau>0$, which relaxes back to $\left|\psi_{\text {ss }}\right\rangle$ as $\tau \rightarrow \infty$. In other words, measuring $g^{(2)}(\tau)$ for $\tau>0$ is directly probing a property (the mean photon number) of the conditional state.

In Ref. [9] we took the next step. That is, rather than simply observing the conditional state prepared by a photodetection, we showed that we could control this state by feedback. That is, by altering the system dynamics subsequent to a photodetection we showed that we could alter the conditional state, and hence change $g^{(2)}(\tau)$ for $\tau>0$. Specifically, we showed that we could freeze the dynamics of the conditional state for some time, making $g^{(2)}(\tau)$ constant, and then release the state to resume its (oscillatory) relaxation to $\left|\psi_{\mathrm{ss}}\right\rangle$. We do this by changing the coherent driving of the cavity at a suitable time $\tau=T$ after the first detection. This technique works only because the system is a quantum one; a classical system consisting of noisy harmonic oscillators cannot be controlled in the same way.

This paper describes in detail our studies of quantum feedback in resonant cavity QED through basic theoretical principles and experimental measurements. The organization of the paper is as follows: Section II covers the theory necessary to understand the experiment: the master equation for the system; quantum trajectories for the system; the quantum feedback algorithm; analogous classical models and why there is no analogous classical feedback algorithm; and, finally, experimental considerations. Section III contains the experimental apparatus and data-taking procedures. Section IV presents the measurements, and section $\mathrm{V}$ concludes.

\section{THEORY}

\section{A. Master Equation}

We study the many atom cavity QED system of Ref. [6]. This system consists of $N$ two-level atoms coupled to a single mode of a Fabry-Perot cavity which is characterized by the field annihilation (creation) operator $\hat{a}$ $\left(\hat{a}^{\dagger}\right)$. The free atom and field Hamiltonians are given by $\hat{H}_{f}=\hbar \omega_{c} \hat{a}^{\dagger} \hat{a}$ and $\hat{H}_{i}=\frac{1}{2} \hbar \omega_{a} \hat{\sigma}_{i}^{z}$ respectively. The transitions between the ground and excited states of the $i$ th atom are described by the standard raising (lowering) Pauli spin operators $\hat{\sigma}_{i}^{\dagger}\left(\hat{\sigma}_{i}\right)$ and $\hat{\sigma}_{i}^{z}=\left[\hat{\sigma}_{i}^{\dagger}, \hat{\sigma}_{i}\right]$. We use the rotating-wave approximation to model the atom-field coupling with the Tavis-Cummings Hamiltonian

$$
\hat{H}_{\mathrm{TC}}=i \hbar \sum_{i} g_{i}\left(\hat{a}^{\dagger} \hat{\sigma}_{i}-\hat{a} \hat{\sigma}_{i}^{\dagger}\right),
$$

where $g_{i}$ is the strength of the coupling between the cavity and the $i$ th atom. For a Gaussian TEM $_{00}$ cavity mode the coupling is:

$$
g_{i}=g \cos \left(k z_{i}\right) \exp \left[-\left(r_{i} / w\right)^{2}\right]
$$

where $r$ is the radial distance from the cavity axis defined by $z, k$ is the magnitude of the cavity field wavevector, $w$ is the waist of the mode, and $g$ is the strength of the maximum coupling given by

$$
g=\mu \sqrt{\frac{\omega_{a}}{2 \hbar \epsilon_{o} V}},
$$

where $\mu$ is the electric dipole moment of the two-level transition and $V$ is the cavity mode volume.

The system is driven by a weak, coherent, laser of frequency $\omega_{0}$ and amplitude $\mathcal{E}$, described by

$$
\hat{H}_{d}=i \hbar\left(\mathcal{E} e^{-i \omega_{o} t} \hat{a}^{\dagger}-\mathcal{E}^{*} e^{i \omega_{o} t} \hat{a}\right) .
$$

We further assume that the cavity QED system is weakly and homogenously coupled to the environment, with the atoms decaying by purely radiative processes. This weak coupling allows for the Born-Markov approximation. Moving to an interaction frame such that the free dynamics of the system and cavity are removed gives the following master equation [10]

$$
\begin{aligned}
\dot{\rho}(t)= & \mathcal{L} \rho(t) \\
= & \mathcal{E}\left[\hat{a}^{\dagger}-\hat{a}, \rho(t)\right]+\sum_{i} g_{i}\left[\hat{a}^{\dagger} \hat{\sigma}_{i}-\hat{a} \hat{\sigma}_{i}^{\dagger}, \rho(t)\right] \\
& +2 \kappa \mathcal{D}[\hat{a}] \rho(t)+\gamma \sum_{i} \mathcal{D}\left[\hat{\sigma}_{i}\right] \rho(t),
\end{aligned}
$$

where

$$
\mathcal{D}[\hat{A}] \rho=\hat{A} \rho \hat{A}^{\dagger}-\left(\hat{A}^{\dagger} \hat{A} \rho-\rho \hat{A}^{\dagger} \hat{A}\right) / 2 .
$$

In Eq. (2.5) the cavity, the atoms, and the driving are all resonant and $\gamma / 2$ and $\kappa$ represent the atom and cavity 
decay rates respectively. We parameterize the strength of the atom-field coupling with respect to these two decay rates with the cooperativity parameter, $C=N C_{1}$ where $C_{1}=g^{2} / \kappa \gamma$. We parameterize the intracavity intensity by the saturation photon number $n_{0}=\gamma^{2} / 3 g^{2}$. We work in the strong-coupling regime with $C_{1}>1$, and $n_{0}<1$.

\section{B. Quantum Trajectories}

It is well known that quantum trajectory theory can be used to describe the evolution of a continuous-in-time monitored open quantum system [10]. In the many-atom cavity QED system we are studying the cavity is the system which is continuously monitored. The arrangement of the measuring apparatus (see Sec. III) is such that the quantum trajectory corresponds to a direct detection unraveling of the master equation [Eq. (2.5)]. In this unraveling a detection has the following effect on the conditional state:

$$
\rho_{1}(t+d t)=\frac{2 \kappa \hat{a} \rho(t) \hat{a}^{\dagger} d t}{P_{1}(t, t+d t)},
$$

where $P_{1}(t, t+d t)=2 \kappa \operatorname{Tr}\left[\hat{a}^{\dagger} \hat{a} \rho(t)\right] d t$ is the probability of getting a detection in the interval $(t, t+d t)$. This change in the system state is commonly referred to as a quantum jump. If no detection is recorded the conditional state is updated by

$$
\rho_{0}(t+d t)=\frac{\tilde{\rho}_{0}(t+d t)}{P_{0}(t, t+d t)}
$$

where $\tilde{\rho}_{0}(t+d t)$ is the unnormalized no-jump conditional state and $P_{0}(t, t+d t)$ is the probability of getting nodetection. Using the fact that the jump and no-jump conditional states must average to give the master equation [Eq. (2.5)], the evolution of $\tilde{\rho}_{0}(t)$ through time is given by

$$
\begin{aligned}
d_{t} \tilde{\rho}_{0}(t)= & \mathcal{E}\left[\hat{a}^{\dagger}-\hat{a}, \tilde{\rho}_{0}(t)\right]+g\left[\hat{a}^{\dagger} \hat{J}_{-}-\hat{a} \hat{J}_{+}, \tilde{\rho}_{0}(t)\right] \\
& +\gamma \sum_{i} \mathcal{D}\left[\hat{\sigma}_{i}\right] \tilde{\rho}_{0}(t)-\kappa\left\{\hat{a}^{\dagger} \hat{a}, \tilde{\rho}_{0}(t)\right\}
\end{aligned}
$$

where $\hat{J}=\sum_{i} \hat{\sigma}$ and $\{\hat{A}, \hat{B}\}$ represents the anticommutator. Here for simplicity we assume that all atoms couple to the cavity with the same strength, $g$.

Since all excitations originate from the driving Hamiltonian, it can be shown that in the weak driving limit $(\mathcal{E} / \kappa$ very small $)$ the probability of a jump is of order $(\mathcal{E} / \kappa)^{2}$ (see Appendix A). Using this it can be shown that to the dominant order in $\mathcal{E} / \kappa$ the normalized nojump conditional state is equal to the unnormalized conditional state. That is $\rho_{0}(t)=\tilde{\rho}_{0}(t)$. Furthermore it can be shown that to the dominant order in $\mathcal{E} / \kappa$ the effect of the $\hat{\sigma}_{i} \rho_{0}(t) \hat{\sigma}_{i}^{+}$terms in Eq. (2.9) are negligible (see Appendix A). This is because the effect of this term on $\rho_{0}(t)$ is an order $\mathcal{E} / \kappa$ greater then the rest of the terms in Eq. (2.9). There exists a pure state factorization of the no-jump conditional state $\left(\rho_{0}(t)=\left|\psi_{0}(t)\right\rangle\left\langle\psi_{0}(t)\right|\right)$ (see Ref. [7]). The time evolution of this pure state is determined by

$$
\begin{aligned}
d_{t}\left|\psi_{0}(t)\right\rangle= & \left(\mathcal{E}\left(\hat{a}^{\dagger}-\hat{a}\right)+g\left(\hat{a}^{\dagger} \hat{J}-\hat{a} \hat{J}^{\dagger}\right)\right. \\
& \left.+\frac{\gamma}{2} \sum_{i} \hat{\sigma}_{i}^{\dagger} \hat{\sigma}_{i}-\kappa \hat{a}^{\dagger} \hat{a}\right)\left|\psi_{0}(t)\right\rangle
\end{aligned}
$$

This has a steady state solution of the form

$$
\begin{aligned}
\left|\psi_{\mathrm{ss}}\right\rangle= & |0, G\rangle+\lambda\left(|1, G\rangle-\frac{2 g \sqrt{N}}{\gamma}|0, E\rangle\right) \\
& +\lambda^{2}\left(\frac{\xi_{0}}{\sqrt{2}}|2, G\rangle+\theta_{0}|1, E\rangle+\frac{\eta_{0}}{\sqrt{2}}|0,2 E\rangle\right) \\
& +O\left(\lambda^{3}\right),
\end{aligned}
$$

where

$$
\begin{aligned}
\xi_{0} & =\frac{\left(1-2 C_{1}^{\prime}\right)(1+2 C)}{1+2 C-2 C_{1}^{\prime}}, \\
\theta_{0} & =-\frac{2 g \sqrt{N}}{\gamma} \frac{1+2 C}{1+2 C-2 C_{1}^{\prime}}, \\
\eta_{0} & =\frac{4 g^{2} \sqrt{N^{2}-N}}{\gamma^{2}} \frac{1+2 C}{1+2 C-2 C_{1}^{\prime}}
\end{aligned}
$$

with $C_{1}^{\prime}=C_{1} /(1+\gamma / 2 \kappa)$. Here $|n, G\rangle$ describes a basis state with $n$ photons in the cavity mode and all atoms in the ground state. The $|E\rangle$ and $|2 E\rangle$ states are the symmetrized one-atom and two-atom excited states respectively. $\lambda$ is the steady state value of the field and is equal to

$$
\lambda=(\mathcal{E} / \kappa) /(1+2 C)
$$

We now make the critical observation that the effect of the term $\hat{a} \rho(t) \hat{a}^{\dagger}$ in Eq. (2.5) is negligible (see Appendix A). The argument is the same as that for neglecting the terms $\hat{\sigma}_{i} \rho(t) \hat{\sigma}_{i}^{+}$in Eq. (2.9). To the dominant order in $\lambda$ the master equation and the non-Hermitian no-jump evolution equation [Eq. (2.10)] are identical. This is also why the waiting time distribution (which is what we actually measure) is proportional to the $g^{(2)}(\tau)$ correlation function in the weak driving limit.

From Eq. (1.3) to calculate $g^{(2)}(\tau)$ we require calculation of the conditional state $\left|\psi_{\mathrm{c}}(\tau)\right\rangle$. This state corresponds to the solution of Eq. (2.10) given the initial condition (a detection at time 0 )

$$
\left|\psi_{\mathrm{c}}(\tau=0)\right\rangle=\hat{a}\left|\psi_{\mathrm{ss}}\right\rangle / \sqrt{\left\langle\psi_{s s}\left|\hat{a}^{\dagger} \hat{a}\right| \psi_{s s}\right\rangle}
$$

Calculating this to order $\lambda$ gives

$$
\left|\psi_{\mathrm{c}}(\tau)\right\rangle=|0, G\rangle+\lambda[\xi(\tau)|1, G\rangle+\theta(\tau)|0, E\rangle],
$$

where the conditioned cavity field evolution, $\xi(\tau)$, and the conditioned atomic polarization field evolution, $\theta(\tau)$, 
are governed by

$$
\begin{aligned}
& d_{\tau} \xi(\tau)=-\kappa \xi(\tau)+g \sqrt{N} \theta(\tau)+\mathcal{E} / \lambda \\
& d_{\tau} \theta(\tau)=-\frac{\gamma}{2} \theta(\tau)-g \sqrt{N} \xi(\tau),
\end{aligned}
$$

where $\xi(0)=\xi_{0}$ and $\theta(0)=\theta_{0}$. That is, the evolution is equivalent to two coupled harmonic oscillators with damping and driving. The quantum effects arise due to the non-classical initial conditions [see Eq. (2.16)]. Without entanglement $\theta_{0}=-2 g \sqrt{N} / \gamma$ the steady state value. The fact that the atomic polarization changes upon detection of the cavity field is evidence of entanglement. The solutions of these equations are both of the general form

$$
f(\tau)=f_{\mathrm{ss}}+e^{-(2 \kappa+\gamma) \tau / 4}\left[A_{i} \cos (\Omega \tau)+B_{i} \sin (\Omega \tau)\right],
$$

where $\Omega=\sqrt{g^{2} N-\left(\frac{1}{2} \kappa-\frac{1}{4} \gamma\right)^{2}}$, and the steady values are $\xi_{\mathrm{ss}}=1$, and $\theta_{\mathrm{ss}}=-2 g \sqrt{N} / \gamma$. The four constants $A_{\xi}, A_{\theta}, B_{\xi}$ and $B_{\theta}$ are given by

$$
\begin{aligned}
& A_{\xi}=\xi_{0}-\xi_{\mathrm{ss}} \\
& A_{\theta}=\theta_{0}-\theta_{\mathrm{ss}} \\
& B_{\xi}=\frac{g \sqrt{N}\left(\theta_{0}-\theta_{\mathrm{ss}}\right)}{\Omega}-\frac{(-\gamma+2 \kappa)\left(\xi_{0}-\xi_{\mathrm{ss}}\right)}{4 \Omega} \\
& B_{\theta}=-\frac{g \sqrt{N}\left(\xi_{0}-\xi_{\mathrm{ss}}\right)}{\Omega}+\frac{(-\gamma+2 \kappa)\left(\theta_{0}-\theta_{\mathrm{ss}}\right)}{4 \Omega} .
\end{aligned}
$$

Using Eqs. (1.3) and (2.17) it is easy to show that $g^{(2)}(\tau)$ is given by

$$
g^{(2)}(\tau)=|\xi(\tau)|^{2}
$$

That is, the correlation function measures the square of the conditioned field amplitude.

\section{Quantum Feedback Control}

In this section we outline the feedback protocol used to capture and stabilize the conditional state. From Eq. (2.17) we note that the evolution of the conditional state (to first order in $\lambda$ ) depends on only the two functions $\xi(\tau)$ and $\theta(\tau)$. This state can be stabilized if we can, via feedback, make $d_{\tau} \xi(\tau)=0$ and $d_{\tau} \theta(\tau)=0$ (put it into a new steady state). From Eqs. (2.18) and (2.19) there are two adjustable parameters that are easily experimentally accessible, the feedback time $T=\tau$ and the driving strength $\mathcal{E}$. The feedback protocol simply consists of applying a different driving strength, $\mathcal{E}^{\prime}$ at certain times $T_{n}$ which satisfy the above conditions. To calculate the feedback times we set $d_{\tau} \theta(\tau)$ in Eq. (2.19) to zero. That is, we choose a time such that the magnitude of the atomic polarization is at a maximum or minimum. This is necessary because changing $\mathcal{E}$ directly affects only $\xi(\tau)$ [Eq. (2.18)], not $\theta(\tau)$ [Eq. (2.19)]. Doing this gives the feedback time constraint

$$
\Omega T_{n}=n \pi+\tan ^{-1}\left[\frac{-A_{\theta}(\gamma+2 \kappa) / 4+B_{\theta} \Omega}{A_{\theta} \Omega+B_{\theta}(\gamma+2 \kappa) / 4}\right],
$$

where $n=0,1,2 \ldots$. For the quantum case (non-classical initial conditions) this can be rearranged to give

$$
\Omega T_{n}=n \pi-\tan ^{-1}\left(\frac{1+2 C}{B_{\theta}^{\prime}+2 C B_{\xi}^{\prime}}\right) .
$$

where $B_{\theta}^{\prime}$ and $B_{\xi}^{\prime}$ are defined as follows

$$
\begin{aligned}
B_{\xi}^{\prime} & =\frac{2 \kappa+\gamma}{4 \Omega} \\
B_{\theta}^{\prime} & =\frac{4 \kappa^{2}-\gamma^{2}-16 \Omega^{2}}{16 \Omega \kappa} .
\end{aligned}
$$

The change in the driving strength is then determined by substituting $\theta\left(T_{n}\right)$ and $d_{\tau} \xi(\tau)=0$ in Eq. (2.18) and solving for $\mathcal{E}$. Doing this gives

$$
\mathcal{E}^{\prime} / \lambda=\kappa \xi\left(T_{n}\right)(1+2 C)
$$

Note we have used the fact that

$$
\theta\left(T_{n}\right)=-2 g \sqrt{N} \xi\left(T_{n}\right) / \gamma
$$

This can be derived from the first condition.

Figure 4 (ii) shows a plot of the evolution of $g^{(2)}(\tau)$ with feedback applied at time $T_{5}$. The details of the plot are explained in Sec. IIE 2.

\section{Semiclassical Dynamics}

As mentioned in the above section Eqs. (2.18) and (2.19) are equivalent to a driven harmonic oscillator, damped at rate $\kappa$, and coupled to a second harmonic oscillator, damped at rate $\gamma / 2$. The strength of the coupling is $g \sqrt{N}$. Thus one may expect that the above feedback protocol for capturing a conditional state is applicable to this semiclassical system. Here we show that this is not the case. To do this we consider three cases, no noise, making a jump by hand, and adding phase noise to the second oscillator.

Before doing this we point out that these coupled harmonic oscillator equations can also be derived from the Maxwell Bloch equations [31, 32]. That is, we assume a semiclassical decorrelation (no-entanglement) between the field and atomic variables: From Eq. (2.5) we find a set of dynamical equations for $\alpha=\langle a\rangle e^{i \omega_{0} t}$, $s_{i}=\left\langle\sigma_{i}\right\rangle e^{i \omega_{0} t}$, and $w_{i}=\left\langle\sigma_{i}^{z}\right\rangle$,

$$
\begin{aligned}
\dot{\alpha} & =-\kappa \alpha+\mathcal{E}+\sum_{i} g_{i} s_{i} \\
\dot{s}_{i} & =g_{i} w_{i} \alpha-\frac{1}{2} \gamma s_{i} \\
\dot{w}_{i} & =-2 g_{i}\left(\alpha^{*} s_{i}+s_{i}^{*} \alpha\right)-\gamma\left(w_{i}+1\right) .
\end{aligned}
$$


where we have again transformed into the interaction frame rotating at the driving laser frequency, $\omega_{0}$.

We assume that all atoms are maximally coupled to the cavity mode, $g_{i}=g$, and that we are in the limit of weak driving where $w_{i}=-1$. Equations (2.32) and (2.33) can then be written in terms of a collective atomic polarization $J=(1 / \sqrt{N}) \sum_{i} s_{i}$

$$
\begin{aligned}
\dot{\alpha} & =-\kappa \alpha+\mathcal{E}+g \sqrt{N} J \\
\dot{J} & =-g \sqrt{N} \alpha-\frac{1}{2} \gamma J
\end{aligned}
$$

which are equivalent to Eqs. (2.18) and (2.19) with $\alpha(t)=\lambda \xi(t)$ and $J(t)=\lambda \theta(t)$.

\section{Coupled Harmonic Oscillators}

The master equation for the system of two coupled harmonic oscillators is

$$
\begin{aligned}
\dot{\rho}(t)= & \mathcal{E}\left[\hat{a}^{\dagger}-\hat{a}, \rho(t)\right]+g \sqrt{N}\left[\hat{a}^{\dagger} \hat{b}-\hat{a} \hat{b}^{\dagger}, \rho(t)\right] \\
& +2 \kappa \mathcal{D}[\hat{a}] \rho(t)+\gamma \mathcal{D}[\hat{b}] \rho(t)
\end{aligned}
$$

where $\mathcal{D}[\hat{A}] \rho$ is defined in Eq. (2.6) and $\hat{b}\left(\hat{b}^{\dagger}\right)$ is the annihilation (creation) operator for the second harmonic oscillator. It is easy to show that in the weak driving limit the steady state for this master equation is pure and takes the form

$$
\begin{aligned}
\left|\psi_{\mathrm{ss}}\right\rangle= & |0,0\rangle+\lambda\left(|1,0\rangle-\frac{2 g \sqrt{N}}{\gamma}|0,1\rangle\right) \\
& +\lambda^{2}\left(\frac{1}{\sqrt{2}}|2,0\rangle-\frac{2 g \sqrt{N}}{\gamma}|1,1\rangle+\frac{4 g^{2} N}{\sqrt{2} \gamma^{2}}|0,2\rangle\right) \\
& +O\left(\lambda^{3}\right)
\end{aligned}
$$

where $|n, m\rangle$ describes the basis state with $n$ excitations in the first oscillator and $m$ excitations in the second. For this system the conditional state (to order $\lambda$ ) after a detection is

$$
\left|\psi_{\mathrm{c}}(0)\right\rangle=|0,0\rangle+\lambda\left(|1,0\rangle-\frac{2 g \sqrt{N}}{\gamma}|1,0\rangle\right),
$$

which is identical to Eq. (2.38) to the same order. Thus the conditional state will not change in time, and the feedback is trivial, in the sense that we already have a stable state so there is no use applying feedback to get a different one. Note this is actually true for all $\mathcal{E}$, as it can be shown, using a $P$-function, that the steady state solution of Eq. (2.37) is

$$
\left|\psi_{\mathrm{ss}}(t)\right\rangle=|\alpha\rangle|\beta\rangle
$$

where $\alpha=\lambda$ and $\beta=-2 g \sqrt{N} \lambda / \gamma$. Acting on these coherent states with a jump operator has no effect.

\section{Coupled Harmonic Oscillators with a Forced Jump}

The second case we consider is when we make a jump by hand. By this, we mean at some time $t=0$ (after the system has reach its original steady state) we apply a delta-pulse to the driving field, thereby forcing the system out of its original steady state. Mathematically this is represented by

$$
\mathcal{E}(t)=\mathcal{E}+\varepsilon \delta(t),
$$

where $\varepsilon$ is the amplitude of the pulse. The effect this has on the cavity fields (driven oscillator) and the atomic fields (second oscillator) initial condition is

$$
\begin{aligned}
& \xi_{0}=\xi_{\mathrm{ss}}+\varepsilon / \lambda \\
& \theta_{0}=\theta_{\mathrm{ss}} .
\end{aligned}
$$

That is, when we force a jump in the cavity field the atomic field is unchanged.

Using these initial conditions, the combined state at time $\tau$ is, to order $\lambda$,

$$
\left|\psi_{\mathrm{c}}(\tau)\right\rangle=|0,0\rangle+\lambda[\xi(\tau)|1,0\rangle+\theta(\tau)|0,1\rangle],
$$

where $\xi(\tau)$ and $\theta(\tau)$ are given by Eq. (2.20) and the four constants $A_{\xi}, A_{\theta}, B_{\xi}$ and $B_{\theta}$ [Eqs. (2.21) - (2.24)] become

$$
\begin{aligned}
& A_{\xi}=\varepsilon / \lambda, \\
& A_{\theta}=0, \\
& B_{\xi}=-\frac{\varepsilon(-\gamma+2 \kappa)}{4 \lambda \Omega}, \\
& B_{\theta}=-\frac{\varepsilon g \sqrt{N}}{\lambda \Omega} .
\end{aligned}
$$

We can now implement a protocol similar to the feedback protocol used in the quantum case. The reason we say only similar is that the protocol does not depend on the conditional state. It is all predetermined; the possible times for changing the driving strength a second time do not depend in any way on measurement results. Thus this is not feedback, but in any case the protocol works as follows. After we first change the driving strength to create non-trivial initial conditions, then at times given by

$$
\Omega T_{n}=n \pi+\tan ^{-1}\left[\frac{4 \Omega}{\gamma+2 \kappa}\right],
$$

where $n=0,1,2, \ldots$ the driving strength is changed again via Eq. (2.30). The possible times for applying the protocol were derived by substituting the above $A_{\theta}$ and $B_{\theta}$ into Eq. (2.26). Note that these times depend on the dynamical parameters of the system, not on the size of the jump. The difference between Eqs. (2.27) and (2.49) is due to the entanglement between the atom and the cavity in the quantum case. Thus an experimental measurement of the difference between these times 

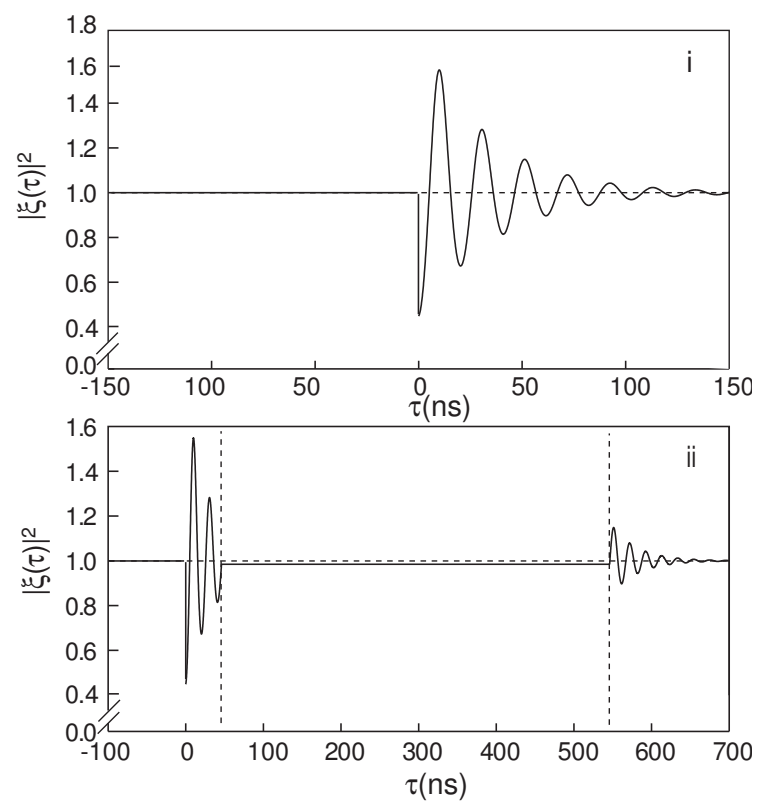

FIG. 1: (i) Time evolution of $|\xi(\tau)|^{2}$ for the semiclassical system when a forced jump is made at $\tau=0$ for $(\Omega, \kappa, \gamma) /(2 \pi)$ $=(48.5,4.9,9.1) \mathrm{MHz}$. (ii) Time evolution of $|\xi(\tau)|^{2}$, for the same parameters as above, showing stabilization at time $T_{4}=$ $46.07 \mathrm{~ns}$ for a duration of $500 \mathrm{~ns}$. The relative change in the driving strength is $\mathcal{E}^{\prime} / \mathcal{E}=0.9922$.

is an implicit measurement of entanglement. For experimental conditions $(\Omega, \kappa, \gamma) /(2 \pi)=(48.5,4.9,9.1) \mathrm{Mhz}$ the semiclassical stabilizing times in nanoseconds are $T_{n}=(n \pi+1.47) /(0.097 \pi)$, which when compared to the quantum case $T_{n}=(n \pi-1.57) /(0.097 \pi)$ gives a difference of $10 \mathrm{~ns}$. These expressions are found by substituting the above parameters, with $g / 2 \pi=\sqrt{3 / 8} 6.1 \mathrm{MHz}$, into Eqs. (2.49) and (2.27). However, because these are periodic times it is equally valid to consider the difference between $T_{n}$ for the quantum case and $T_{n}-1$ for the semiclassical case. Doing this gives a time difference of only 0.33 ns. This would be a much harder difference to measure experimentally, as our binning is $0.5 \mathrm{~ns}$, and is within the experimental error of this work.

Figure 1 i shows the $|\xi(\tau)|^{2}$ correlation function for this classical system when we have forced by hand a jump at $t=0$. Here we see that the system relaxes back to its original steady state. We have chosen the amplitude of the jump $\varepsilon$ such that the initial value for $\xi(\tau), \xi_{0}$, in both the quantum case and semiclassically case are identical. This choice is arbitrary as it does not effect the semiclassical stabilizing times. Fig. 1 ii shows $|\xi(\tau)|^{2}$ for the case when the driving strength is changed via Eq. (2.30) at time $T_{4}=46.07 \mathrm{~ns}$. The original steady state is recovered at a later time by returning the driving strength to its previous value. The duration of the changed driving strength is $500 \mathrm{~ns}$. This is represented by the vertical dotted lines.

\section{Coupled Harmonic Oscillators with Phase Diffusion}

To consider genuine feedback in a semiclassical system we have to introduce noise into the system, so that the conditional state changes upon detection. However, we still want all the excitations to come from the driving, so we can only include phase noise. To keep a close analogy with the quantum case we want to keep the first harmonic oscillator (the cavity) noiseless. Therefore we introduce the phase noise into the second ('atomic') harmonic oscillator. This corresponds to adding the following term to the master equation [Eq. (2.37)]

$$
\Gamma \mathcal{D}\left[\hat{b}^{\dagger} \hat{b}\right] \rho(t) .
$$

The effect of this is that the steady state can no longer be assumed pure (see appendix B). However, the no-jump evolution, in the weak driving limit, is still equal to the master equation evolution. We remind the reader that by 'no-jump' we mean no-detections of photons from the cavity. Thus a measurement of $g^{(2)}(\tau)$ is still proportional to the waiting time distribution. In appendix B we show that the conditional state at time $\tau$ (after a detection at time $\tau=0$ ) to second order in $\lambda$ is

$$
\begin{aligned}
\rho_{c}(\tau)= & |0,0\rangle\langle 0,0|+\lambda^{2}\left[s_{5}(t)|0,1\rangle\langle 1,0|+\text { H.c }\right] \\
& +\lambda\left[f_{1}(t)|0,0\rangle\left\langle 0,1\left|+f_{2}(t)\right| 0,0\right\rangle\langle 1,0|+\text { H.c }\right] \\
& +\lambda^{2}\left[s_{4}(t)|0,1\rangle\left\langle 0,1\left|+s_{6}(t)\right| 1,0\right\rangle\langle 1,0|\right] .(2.51)
\end{aligned}
$$

Here $f_{1}(t), f_{2}(t), s_{4}(t), s_{5}(t)$, and $s_{6}(t)$ is our notation for first $\left(f_{i}\right.$ 's) and second $\left(s_{i}\right.$ 's) order terms in $\lambda$. From appendix $\mathrm{B}$ the evolution of these parameters through time is given by

$$
\begin{aligned}
d_{\tau} f_{1}(\tau)= & -(\gamma+\Gamma) f_{1}(\tau) / 2-g \sqrt{N} f_{2}(\tau) \\
d_{\tau} f_{2}(\tau)= & -\kappa f_{2}(\tau)+g \sqrt{N} f_{1}(\tau)+\mathcal{E} / \lambda \\
d_{\tau} s_{4}(\tau)= & -\gamma s_{4}(\tau)-g \sqrt{N}\left[s_{5}(\tau)+s_{5}^{*}(\tau)\right], \\
d_{\tau} s_{5}(\tau)= & -(\kappa+\gamma / 2+\Gamma / 2) s_{5}(\tau)+g \sqrt{N}\left[s_{4}(\tau)\right. \\
& \left.-s_{6}(\tau)\right]+(\mathcal{E} / \lambda) f_{1}^{*}(\tau) \\
d_{\tau} s_{6}(\tau)= & -2 \kappa s_{6}(\tau)+g \sqrt{N}\left[s_{5}(\tau)+s_{5}^{*}(\tau)\right] \\
& +(\mathcal{E} / \lambda)\left[f_{2}^{*}(\tau)+f_{2}(\tau)\right]
\end{aligned}
$$

Note Eqs. (2.52) and (2.53) are equivalent to (2.19) and (2.18) with $\gamma \rightarrow \gamma+\Gamma$. For this system the $g^{(2)}(\tau)$ correlation function is given by

$$
g^{(2)}(\tau)=\frac{\operatorname{Tr}\left[\hat{a}^{\dagger} \hat{a} \rho_{\mathrm{c}}\right]}{\operatorname{Tr}\left[\hat{a}^{\dagger} \hat{a} \rho_{\mathrm{ss}}\right]}=\frac{s_{6}(\tau)}{s_{6}^{\mathrm{ss}}} .
$$

To capture the conditional state of the above semiclassical system we need to be able to set all the derivatives in Eqs. $(2.52)-(2.56)$ to zero. Thus the problem is there are five equations to control and only two adjustable parameters; feedback time $T$ and driving strength $\mathcal{E}$. However we can ask the following question, using the same feedback protocol as used in the quantum case [stabilizing $f_{1}(\tau)$ and $f_{2}(\tau)$ ] what effect does non-stabilization 

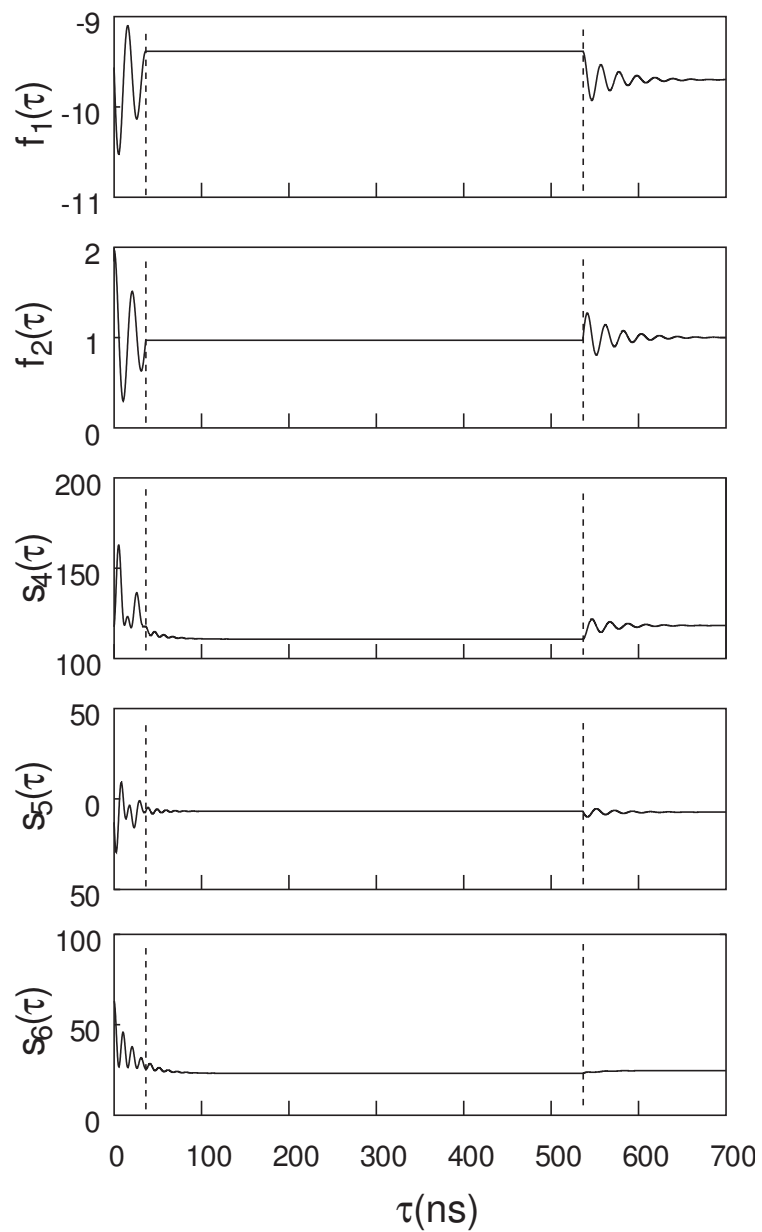

FIG. 2: Time evolution of $f_{1}(\tau), f_{2}(\tau), s_{4}(\tau), s_{5}(\tau)$, and $s_{6}(\tau)$ for $(\Omega, \kappa, \gamma, \Gamma) /(2 \pi)=(48.5,4.9,6.0,4.0)$ Mhz. Feedback is applied at time $T_{4}=36.21 \mathrm{~ns}$ and the duration of the feedback pulse is represented by the vertical dotted lines (500ns). The relative change in the driving strength is $\mathcal{E}^{\prime} / \mathcal{E}=0.9675$

of $s_{4}(\tau), s_{5}(\tau)$, and $s_{6}(\tau)$ have on $g^{(2)}(\tau)$ ? Here we do not go through the details again as $f_{1}(\tau)$ and $f_{2}(\tau)$ are equivalent to $\theta(\tau)$ and $\xi(\tau)$ respectively under the transformation $\gamma \rightarrow \gamma+\Gamma$. Thus the feedback must be applied at times given by Eq. (2.26). The two constants $A_{\theta}$ and $B_{\theta}$ are found by substituting the above initial conditions into Eqs. (2.22) and (2.24) respectively. Note in deriving these constants and the times we must also make the transformation $\gamma \rightarrow \gamma+\Gamma$.

Figure 2 shows the application of the feedback protocol. Here we observe that when we implement the feedback at time $T_{4}, f_{1}(\tau)$ and $f_{2}(\tau)$ are stabilized, but $s_{4}(\tau)$, $s_{5}(\tau)$, and $s_{6}(\tau)$ are far from being stabilized. The effect of not been able to stabilize these parameters on $g^{(2)}(\tau)$ is shown in Fig. 3. Here we observe that the correlation function is not frozen in the desired way at all. When the feedback is turned off $g^{(2)}(\tau)$ relaxes back to one.

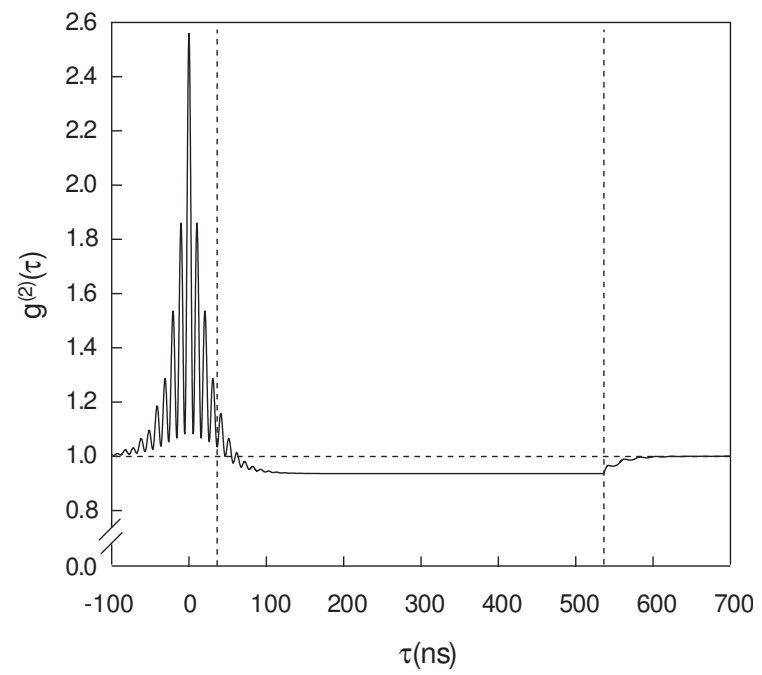

FIG. 3: $g^{(2)}(\tau)$ for $(\Omega, \kappa, \gamma, \Gamma) /(2 \pi)=(48.5,4.9,6.0,4.0)$ $\mathrm{MHz}$, the same parameters as in Fig. 2.

\section{E. Experimental Considerations}

Our experiment works with a highly collimated beam of atoms traversing the mode of an optical resonator, in contrast with the fixed-atom efforts currently under way [33-36]. Our work with conditional field [29] and conditional intensity measurements [28] shows that we can still observe the quantum fluctuations in the light with an atomic beam because in the weak-field limit the atoms act as a single collective entity (see Eq. (2.35) and Eq. (2.36)).

\section{Random Distribution of the Atoms}

We improve the quantum predictions from the maximally coupled atom using the random distribution of atoms in the $\mathrm{TEM}_{00}$ cavity mode of Sanders and Carmichael [37]. We then use it evaluate the modified $A_{\xi}$ for a random distribution of atom-field couplings of Rempe et al. [38]. Our approach is to couple an atom to the cavity according to the distribution and repeat for $N$ atoms. The details of this calculation are reported elsewhere [39]. For now we simply report the final result for $\overline{A_{\xi}}$ which is $A_{\xi}$ ensemble averaged over a random distribution of atoms throughout the cavity mode. In the limit of large atomic beam density $C / C_{1} \gg 1$ we find

$$
\overline{A_{\xi}}=-\frac{3}{4} C_{1}^{\prime},
$$

which we compare with the $N$-identically coupled case in the same limit

$$
A_{\xi}=-2 C_{1}^{\prime} .
$$

The size of the effect still scales as $C_{1}$ but is diminished by more than a factor or two. This calculation 
assumes that all the atoms are resonant, which is not the case for an atomic beam with imperfect collimation. We have found that a small Doppler detuning, that allows excitation of the two quadratures of the electromagnetic field and polarization, reduces any non-classical features of the field [28].

The choice of atomic transition and the geometry of the cavity fix the optimal coupling $g$. We scale the optimal coupling $g$ by $\sqrt{3 / 8}$ to take into account the random distribution of atoms. The comparison between theory and experiments requires that we fix the frequency of the Rabi oscillations, which is proportional to $g \sqrt{N}$. We use the scaled $g$ and the value of $N$ obtained from low intensity counting rates with and without atoms to match the experimental oscillation frequency and the theoretical parameters.

\section{Homogeneous Transit Broadening}

To model this we consider one of the methods used by Clemens and Rice [40]. They did a quantum trajectory simulation in which the number of atoms in the cavity was assumed constant, but at random times one atom was removed and replaced by an atom in the ground state. The times were chosen from an approximately Gaussian distribution with a mean and width of $1 / \gamma_{T}$. This removal and replacement was effected by measuring the atomic state of one of the atoms. If it was found to be in the excited state, then it is lowered; if in the ground state, it is left unchanged. That is, if the $i$ th atom is affected then the state is acted upon by the superoperator $\mathcal{R}_{i}$ defined as

$$
\mathcal{R}_{i} \rho=\hat{\sigma}_{i} \rho \hat{\sigma}_{i}^{\dagger}+\left|g_{i}\right\rangle\left\langle g_{i}|\rho| g_{i}\right\rangle\left\langle g_{i}\right| .
$$

If instead of assuming a Gaussian distribution for the transit times, we assume a negative exponential distribution with same mean and width, then the removal and replacement process becomes Poissonian with rate $\gamma_{T}$. This means it can be modeled by a master equation

$$
\mathcal{L}_{\text {transit }}=\gamma_{T} \sum_{i}\left(\mathcal{R}_{i}-1\right) \text {. }
$$

This can be rewritten in Lindblad form as

$$
\mathcal{L}_{\text {transit }}=\gamma_{T} \sum_{i}\left(\mathcal{D}\left[\hat{\sigma}_{i}\right]+\mathcal{D}\left[\hat{\sigma}_{i}^{z} / 2\right]\right) .
$$

The effect of this on the longitudinal and transverse decay rates of each atom is

$$
\begin{aligned}
\gamma_{\|} & =\gamma+\gamma_{\mathrm{T}}, \\
\gamma_{\perp} & =\gamma / 2+\gamma_{\mathrm{T}} .
\end{aligned}
$$

As discussed in Sec. IID 3, the addition of a dephasing term like $\mathcal{D}\left[\hat{\sigma}^{z}\right]$ spoils the purity of the steady state in the weak driving limit, and invalidates the feedback algorithm. In Ref. [28] the transit time was measured to be approximately 100ns, giving $\gamma_{\mathrm{T}} / 2 \pi \approx 1.6 \mathrm{MHz}$. Half of this contribution to the transverse decay rate, that is $\left(\gamma_{\mathrm{T}} / 2\right) / 2 \pi \approx 0.8 \mathrm{MHz}$ comes from the problem (dephasing) term. However, this is less than $10 \%$ of the total amplitude decay rate for the experimental system system, which is

$$
\left(\kappa+\gamma_{\perp}\right) / 2 \pi \approx 9.5 \mathrm{MHz}
$$

It seems reasonable to assume that the mixing induced by this term is small (and the experiments bear this out). Rather than simply discarding the term, however, we instead replace it by $\mathcal{D}[\hat{\sigma}]$, since this gives the same contribution to $\gamma_{\|}$, which is the most important rate for determining the shape of $g^{(2)}(\tau)$. Thus in the final analysis, we modelled transit broadening simply by the replacement

$$
\gamma / 2 \pi \rightarrow\left(\gamma+2 \gamma_{T}\right) / 2 \pi \approx 9.2 \mathrm{MHz}
$$

Note that this corrects the expression used in Eq. (2.66) in Ref. [39].

Figure 4 (i) illustrates the resulting conditional intensity $g^{(2)}(\tau)$ expected in the experiment with the additional corrections. The random distribution of atoms produces a corrected coupling constant $g / 2 \pi$ changing from $6.1 \mathrm{MHz}$ to $\sqrt{3 / 8} 6.1 \mathrm{MHz}$. The Rabi frequency is $\Omega / 2 \pi=48.5 \mathrm{MHz}$.

Figure 4 (ii) shows a plot of the normalized conditioned intensity evolution, $g^{(2)}(\tau)$ with feedback. The application of the feedback step is at a time given by Eq. (2.27) $\left(T_{5}=46.4 \mathrm{~ns}\right)$ at the third oscillation $(n=5)$ following the photon arrival at $\tau=0$. This forces the system into a new steady state. The former oscillatory state is recovered at a later time (+500 ns), when the feedback stops, with the exact same amplitude and phase that it had.

\section{Optimal Coupling}

Ideally we would like to force the system into any state with the application of feedback; however our protocol limits and sets conditions for what is possible. We could make the initial step $\left|A_{\xi}\right|$ Eq. (2.21), originating from the first escape of a photon, as large as possible to maximize the feedback effect. This is done by increasing the atom-field coupling, $g$. There are two drawbacks to this approach. The first is that the output flux scales as $g^{-4}$, and the data taking time becomes prohibitively long. The second problem is that increasing $g$ only increases the size of the overall fluctuation, not the relative size of the feedback that must be applied. We should apply the feedback that will change the state by the largest amount with respect to the initial field fluctuation. This is equivalent to maximizing the following function

$$
\Xi\left(T_{n}\right)=\frac{\xi\left(T_{n}\right)-1}{A_{\xi}},
$$

where $T_{n}$ is given by Eq. (2.27). Fig. 5 is a plot of $\Xi$, using typical parameters for our experiment. It qualita- 

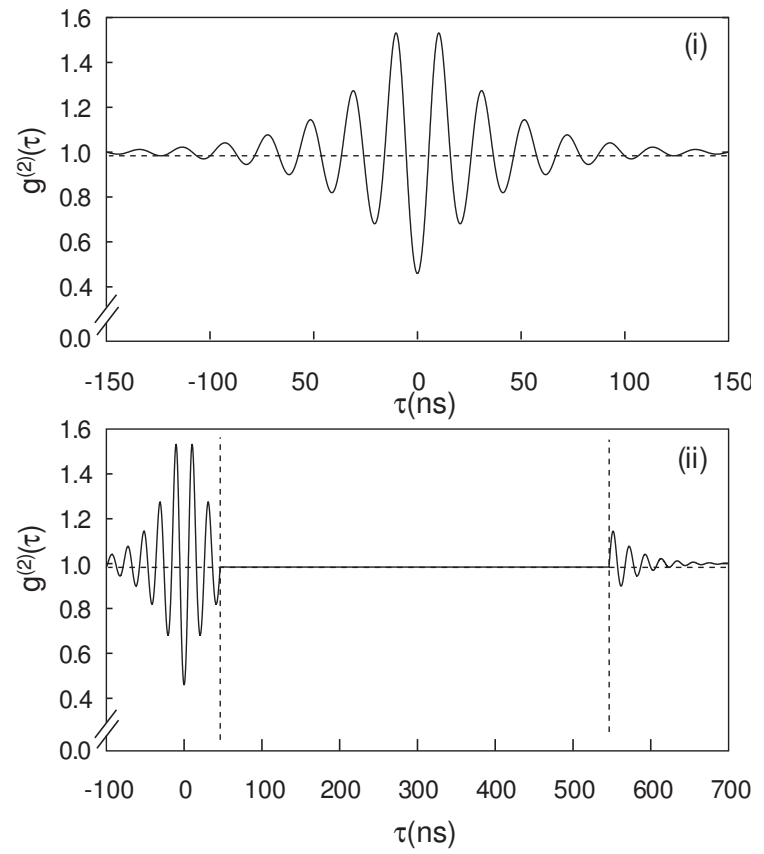

FIG. 4: (i) Time evolution of $g^{(2)}(\tau)$ for $(\Omega, g, \kappa, \gamma) / 2 \pi=$ $(48.5, \sqrt{3 / 8} 6.1,4.9,9.1) \mathrm{MHz}$. (ii) Time evolution of $g^{(2)}(\tau)$, for the same parameters as above, showing stabilization into a new steady state at time $T_{5}=46.4 \mathrm{~ns}$ for a duration of $500 \mathrm{~ns}$. The relative change in the driving strength $\mathcal{E}^{\prime} / \mathcal{E}=0.9924$

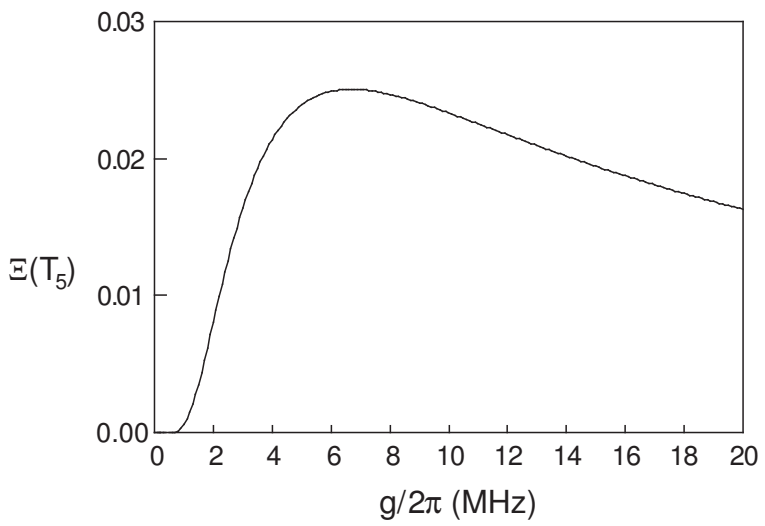

FIG. 5: Fractional change in the field as a result of the feedback applied for $n=5$ in Eq. $(2.27) .(\kappa, \gamma) /(2 \pi)=(4.9,6.0)$ $\mathrm{MHz}$ and $N=100$ atoms identically coupled.

tively shows that there is an ideal value for $g$ with this feedback proposal.

\section{APPARATUS}

We briefly review the apparatus and refer the reader to the more explicit discussions in Ref. [28]. The apparatus consists of an optical Fabry-Perot cavity, a thermal beam of ${ }^{85} \mathrm{Rb}$, a cw excitation laser, a detection system, and feedback equipment. Fig. 6 shows the primary compo-

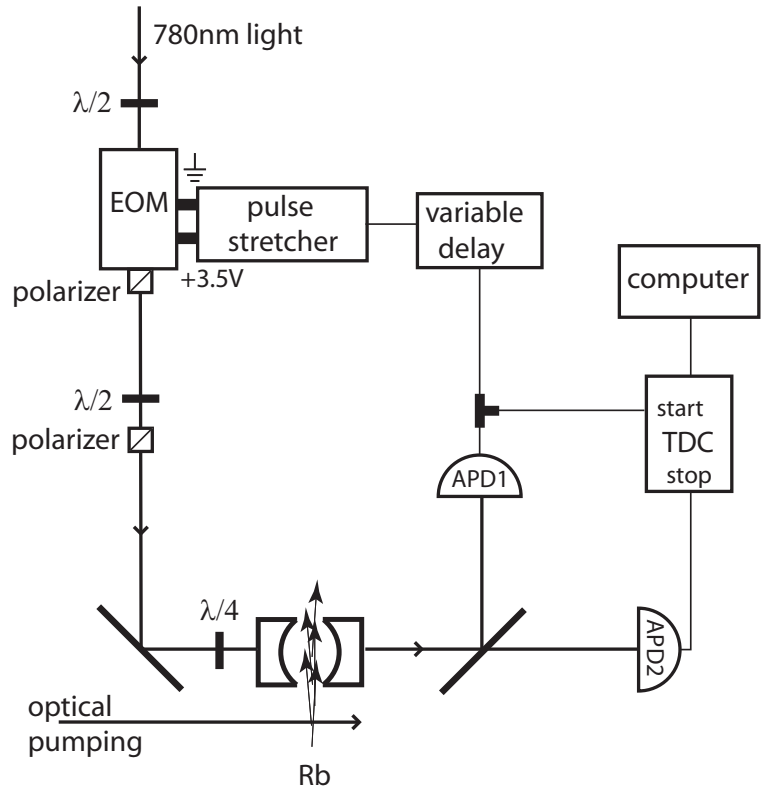

FIG. 6: Simplified diagram of the experimental setup. Two Avalanche Photo-Diodes (APD)measure the intensity for the correlator. Gating electronics, a time-to-digital converter (TDC), and a histogramming memory and computer process the correlation. Photo-detections at APD1 trigger a change in the intensity injected into the cavity via an electro-opticmodulator (EOM). Optics shown are relevant for control of the size of the intensity step, and the polarization of the light injected into the cavity.

nents in the apparatus. The three frequencies in the system, the atomic transition, cavity resonance, and driving laser are all equal, $\omega_{\mathrm{a}}=\omega_{\mathrm{c}}=\omega_{\ell}$, unless explicitly stated otherwise. The excitation laser drives the coupled atomscavity system from one side. The information collected at the other side triggers a fast optical feedback routine which alters the dynamics of the system. We use the same collected light to make intensity correlation measurements.

\section{A. Cavity QED System}

The cavity defines a $\mathrm{TEM}_{00}$ mode with two mirrors with different transmission coefficients, $T_{1}=15 \mathrm{ppm}$ and $T_{2}=300 \mathrm{ppm}$. The input transmission is smaller than the output to ensure that most of the signal escapes from the cavity on the detector side. A typical cavity finesse for this arrangement is $\mathcal{F} \approx 21000$. The cavities for these measurements vary in size between $600-900 \mu \mathrm{m}$ long. Both mirrors have a radius of curvature of $50 \mathrm{~mm}$. The cavity waists are between $30-45 \mu \mathrm{m}$. The cavities give us coupling constants in the range: $4.5<g / 2 \pi<6.5$ $\mathrm{MHz}$.

Two cylindrical piezo-electric-transducers (PZT) control the length of the cavity. A Pound-Drever-Hall alternating lock controls the cavity length. The lock and 
signal beams have orthogonal linear polarization when combined at a beam splitter before the cavity entrance. Before we inject the lock into the cavity it travels through a chopping wheel with two different sets of apertures, which alternately blocks the beam for $350 \mu \mathrm{s}$, and passes it for $600 \mu \mathrm{s}$. A polarizing beamsplitter sends the output signal through the same chopping wheel at a different place and then to the intensity detectors of the correlator, the wheel blocks the output of the cavity when the lock enters and protects the sensitive photo-detectors. The transmitted lock travels to a photomultiplier, where we monitor the quality of the cavity lock.

The lock and signal beams are both derived from a Coherent Verdi-5 pumped Ti:Sapph 899-01. We use part of the laser output to lock the laser with a Pound-DreverHall technique. An acousto-optic modulator (AOM) adjusts the signal and lock beam frequencies around the $5 S_{1 / 2}, F=3, m_{F}=3 \rightarrow 5 P_{3 / 2}, F^{\prime}=4, m_{F}^{\prime}=4$ transition of ${ }^{85} \mathrm{Rb}$ at $780 \mathrm{~nm}$ with $\gamma / 2 \pi=6.07 \mathrm{MHz}$.

An oven located $35 \mathrm{~cm}$ from the cavity produces a thermal beam of $\mathrm{Rb}$ atoms with $T \approx 450 \mathrm{~K}$. A computer controlled feedback loop maintains the temperature to within $\pm 0.1 \mathrm{~K}$. Several slits, located between the oven and cavity, collimate the atomic beam. The final slit collimates the transverse profile of the atomic beam to a spread of $2.8 \mathrm{mrad}$.

A diffusion pump and a liquid nitrogen cooled copper sleeve produce a typical operating pressure of about $5 \times 10^{-7}$ Torr at a gauge located away from the interaction region. The atomic beam may generate a large amount of background $\mathrm{Rb}$ in the region of the cavity. The copper sleeve surrounds the cavity and, when liquid nitrogen cooled, dramatically reduces this background.

A 5 Gauss magnetic field which is collinear to the cavity axis to within $40 \mathrm{mrad}$ provides the quantization axis. Atoms intersect with a $2 \mathrm{~mm}$ diameter, $30 \mathrm{~mW} / \mathrm{cm}^{2}$, circularly polarized optical pumping beam $1 \mathrm{~cm}$ before entering the cavity. The atoms are prepared into the $5 S_{1 / 2}, F=3, m_{F}=3$ magnetic sublevel by repeated excitations to the $F^{\prime}=4$ state with the circularly polarized light.

\section{B. Correlator}

Two EG\&G avalanche photodiodes (APD) record photodetections for the intensity correlations. The start detector is a model SPCM-AQ-151 and stop detections are from a model SPCM-AQR-12. The detectors are at the output of the cavity, behind a 50/50 beam splitter.

A $g^{(2)}(\tau)$ measurement calls for the entire emitted photocurrent record of a particular source. One then histograms the time between every possible pair of photodetections in the record [41]. A time-to-analog-convertor (TAC) or time-to-digital-convertor (TDC) measurement in which individual pairs of start and stop photons are recorded, however, provides the distribution of waiting times between events. For sufficiently small count rates,

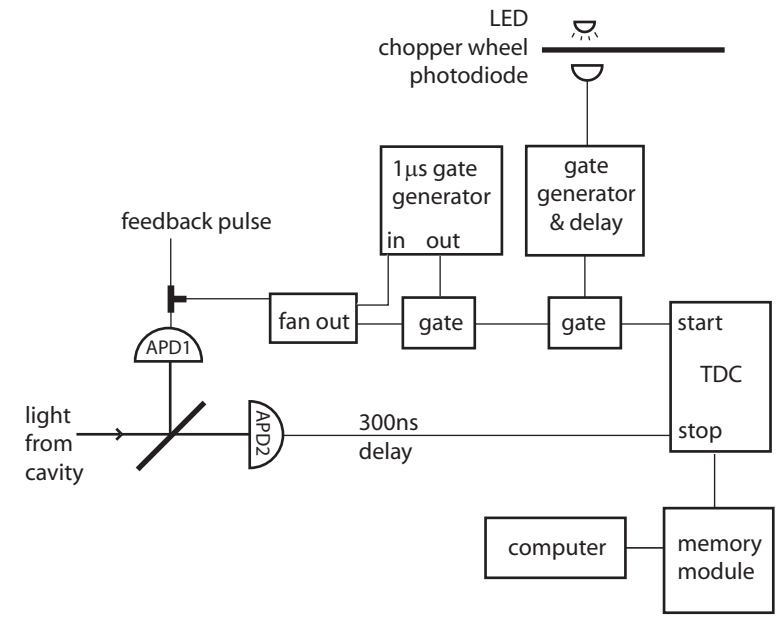

FIG. 7: Simplified diagram of correlation electronics. Electronic pulses produced by APD1 are split and used to initiate optical feedback and to start a TDC. The start pulses pass though two gates which ensures that the photon initiating an event did not originate from the cavity locking beam, and prevents the TDC from re-starting.

however, the waiting time distribution is proportional to $g^{(2)}(\tau)[42,43]$ (see discussion in Sec. II B).

The detectors are in a dark box, to minimize the background count rate, which is typically less than $500 \mathrm{~Hz}$. The APDs produce a large amount of broad band light after every photodetection which produces crosscorrelations between the detectors [44]. Located immediately before each APD to block this light is an optical bandpass filter with $85 \%$ transmission at $780 \mathrm{~nm}$, and a $10 \mathrm{~nm}$ spectral width. The APDs produces transistortransistor logic (TTL) pulses which propagate through a series of nuclear inline module (NIM) modules. A LeCroy model 3377 TDC records the time between photodetections in the two APDs, and a computer histograms the results.

Photodetections in APD1 define $\tau=0$. Fig. 7 is a block diagram of the correlation electronics. A power splitter (EG\&G MT-050) splits them and sends one copy, for feedback, to an EG\&G DB-263 variable delay box. The other is sent to a LeCroy 688AL level adapter which produces a NIM pulse from the TTL input. A LeCroy $428 \mathrm{~F}$ linear fan-out produces copies of the NIM pulse for gating electronics. Once a timing interval has begun, all photodetections from that detector are ignored for a $1 \mu$ s period. The NIM pulse is gated to pass only when the chopping wheel is blocking the cavity lock beam. The NIM pulse is converted to an ECL pulse with a Phillips Scientific 7126 level translator and becomes the start pulse of the TDC. The TTL proceeds to a Stanford Research model SR400 photon counter module, which counts the APD photodetections and sends the count rates to a computer.

Photodetections from APD2 are the stop pulses for the 
TDC. Once the TDC receives a start it records up to 16 stops in the next $1 \mu$ s and sends the event to a LeCroy model 4302 memory module which stores up to 16,000 events. When the memory is full, it sends the events to a computer.

Although up to 16 stops per start may be stored in the memory, events with more than one stop are quite rare. We typically operate with a mean intra-cavity photon number of $n \approx 0.03$. Fewer than $0.5 \%$ of the time are there two excitations in the cavity, which are required to have both a first and a second photon.

The feedback information is derived from the TTL produced by APD1. The TTL from the start detector, after proceeding through the power splitter and variable delay, reaches a pulse stretching circuit. The heart of this circuit is a fast Linear Technology comparator, the LT1720, which has a 4.5 ns response time, with a jitter of $+15 \mathrm{ps}-11 \mathrm{ps}$. The APD TTL is a short $10 \mathrm{~ns}$ pulse, which drives the pulse stretching circuit. After a short delay, the circuit produces a longer pulse. A timing capacitor determines the length of this pulse. We have tested the circuit to produce pulses from $100 \mathrm{~ns}$ to $5 \mu \mathrm{s}$ in length. The output of the circuit drives a Gsänger model LM0202P-IR5W electro-optic-modulator (EOM) with an output polarizer attached. The application of the voltage to the EOM/polarizer pair produces an optical intensity step.

The supply voltage of the comparator fixes the amplitude of the output of the pulse stretcher to $3.5 \mathrm{~V}$. The pulse stretcher always applies the same voltage to the EOM. Adjusting the amplitude of the intensity step is done optically.

The half wave voltage of this EOM is a few hundred volts, which means that the EOM should not appreciably alter the phase of the transmitted light when $V_{i}=3.5 \mathrm{~V}$ is applied. We verified this by inserting the EOM into a Mach-Zehnder interferometer, and observing the change in the fringe transmitted at one output port when $V_{i}$ was applied. This noise-limited measurement reveals that the EOM alters the phase of the transmitted light by less than 30 mrad.

The earliest time following a photodetection that we can alter the intensity of the light entering the cavity is determined by a combination of electronic and optical delays. There are 8 ns of optical propagation between the cavity and APDs. The APDs themselves have been measured to have an internal delay of $18 \mathrm{~ns}$ [45]. There are 9 ns of coaxial cable delay between the APD and the pulse stretcher, the pulse stretcher has an internal delay of about $5 \mathrm{~ns}$. The stretcher is directly on top of the EOM, and then there are another $3 \mathrm{~ns}$ of optical path between the EOM and the cavity. The total delay is $43 \mathrm{~ns}$.

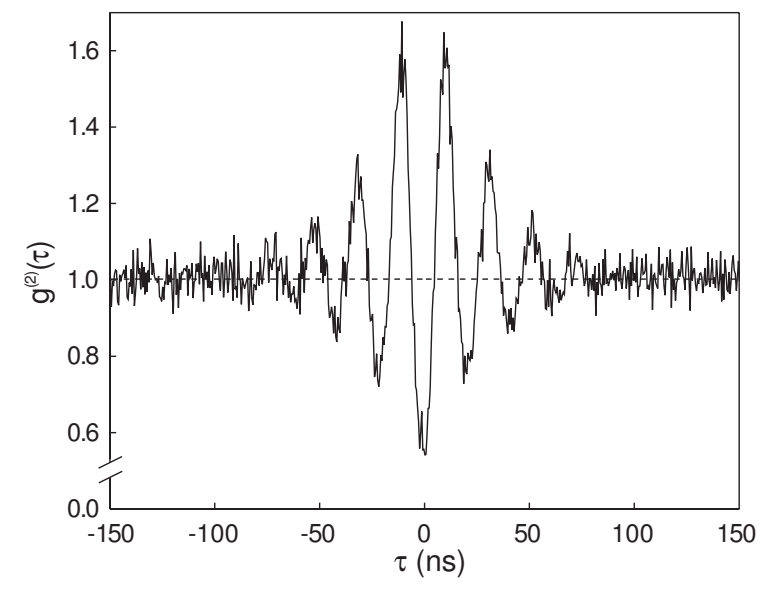

FIG. 8: Measured $g^{(2)}(\tau) . \quad \tau=0$ is defined by a photodetection in APD1. $(\Omega, g, \kappa) / 2 \pi=(48.5,6.1,4.9) \mathrm{MHz}$. Data binned into $1.0 \mathrm{~ns}$ bins.

\section{EXPERIMENT}

We begin with a stabilized oven temperature and measure the intensity correlation $g^{(2)}(\tau)$ to obtain the oscillation frequency, the vacuum Rabi frequency, $\Omega$.

The data taking routine begins by maximizing the nonclassicality of the signal at $\tau=0$. We focus on the subPoissonian character. If the atomic beam is not perpendicular to the cavity mode, the Doppler shifted atoms will be detuned from the cavity resonance. The transmission is a minimum on resonance and we adjust the frequency of the cavity drive to this minimum. The transmission is a broad function of detuning, and this procedure allows us to set the detuning to within $1 \mathrm{MHz}$ of resonance. We then take several correlation measurements in this frequency region to find the optical frequency that minimizes $g^{(2)}(0)$.

Figure 8 shows a typical $g^{(2)}(\tau)$ measurement, note the qualitative agreement with the model prediction shown in Fig. 4 (i). A photon is emitted from the cavity at $\tau=0$, disturbing the steady state of the system. The system then exchanges any remaining energy between the cavity mode and atoms at the coupling frequency, $\Omega$. At any time during this exchange, the system can lose this excitation through either atomic spontaneous emission or through a cavity emission. Without a feedback step, our driven, coupled system again resides in the steady state after some characteristic time dictated by an average of the loss rates, $\frac{1}{2}\left(\kappa+\frac{\gamma}{2}\right)$. This evolution can be seen in the correlation function in Fig. 8. The data is normalized by the average number of counts received at long time (after the correlation has decayed away). This correlation is sub-Poissonian $\left(g^{(2)}(0)=0.48 \pm .02\right)$, antibunched (positive curvature at $\tau=0$ ), and has a deviation from unity at a later time $\tau$ that is larger than the initial deviation from unity at $\tau=0$. We extract from the data set the vacuum Rabi frequency $\Omega$ with typical uncertainties of less than $10 \%$. The coupling constant $g$ comes from the 
known geometry of the cavity and has an uncertainty of less than $5 \%$, while the decay rate of the cavity $\kappa$ comes from the measured linewidth of the cavity mode with uncertainties of less than $5 \%$.

\section{A. Correlation measurements with quantum feedback}

Once the $g^{(2)}(0)$ is as low as possible, we apply feedback to modify the conditional intensity with the protocol defined in Section II C. This produces a non-symmetric correlation function. Notice that our detection scheme allows us to observe $\tau<0$ correlations. Since the $g^{(2)}(\tau)$ function is time-symmetric by construction we use the negative times as a reference to see how feedback has altered the positive $(\tau>0)$ side. Typically we choose to feed back at the earliest time after the experimental minimal feedback time of 43 ns. A first guess feedback pulse with an approximated amplitude modifies the evolution of the system. To estimate if the size of the intensity-step is correct, we compare the size of the restored oscillation to the oscillation identified for suppression. The restored energy exchange should be the same magnitude as the frozen exchange. Once the correct amplitude is established, we determine the feedback time by examining the new steady state the system resides in after the intensity step. The delay of the step is then adjusted so that the value of the intensity correlation is equal to the rate of photo-emission in the new steady state.

Figure 9 shows an intensity correlation corresponding to a feedback intensity step of length $500 \mathrm{~ns}$, $(\Omega, g, \kappa, \gamma) / 2 \pi=(48.5 \pm 3,6.1 \pm 0.3,4.9 \pm 0.2,9.1) \mathrm{MHz}$ The evolution of the system has been stopped with an intensity step $\Delta I=-2.7 \%$ applied at $\tau_{\mathrm{fb}}=45 \mathrm{~ns}$ in agreement with Eq. (2.27) which gives, with our uncertainties $46.4 \pm 3$ ns. A dashed line marks the original steady state value of the system, this new steady state is clearly below the original. At the end of the intensity step, the drive is returned to its original value. The resulting oscillation in the system is of the same amplitude and phase as the suspended oscillation, as can been seen by comparing the positive and negative times of Fig. 9.

It is also possible to extend the correlation time of the energy exchange by moving the intensity driving the system further from the steady state value. Fig. 10 shows a case where the intensity step up and return are timed so that the system continues to oscillate beyond its natural correlation time, although the decay inherent to the system is visible between the step up and return. In this case $\Delta I=+3 \%$, applied at $\tau_{\mathrm{fb}}=56 \mathrm{~ns}$, and the drive is returned to its original value after $120 \mathrm{~ns}$.

Figure 11 demonstrates that it is also possible to stop the evolution of the system at a photo-emission rate higher than the original steady state. Here the intensity change required is smaller, $\Delta I=+0.5 \%$, applied at $\tau_{\mathrm{fb}}=43 \mathrm{~ns}$. As a result, the new steady state of the system is very close to the original value. The gray box

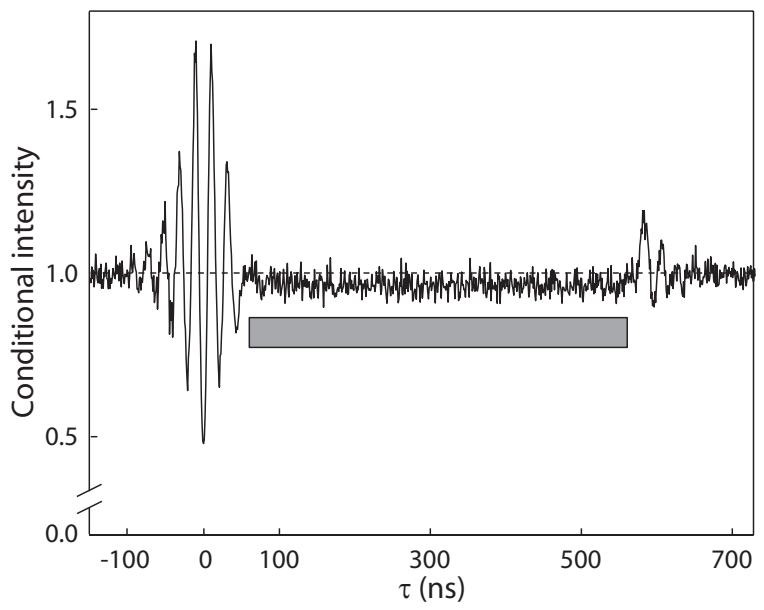

FIG. 9: Measured intensity correlation function. $(\Omega, g, \kappa) / 2 \pi=(48.5,6.1,4.9) \mathrm{MHz}$. The grey box indicates the application time of the square feedback pulse, $\Delta I=-2.7 \%, \tau_{\mathrm{fb}}=45 \mathrm{~ns}$, the intensity step was $500 \mathrm{~ns}$ long. Data binned into $1.0 \mathrm{~ns}$ bins.

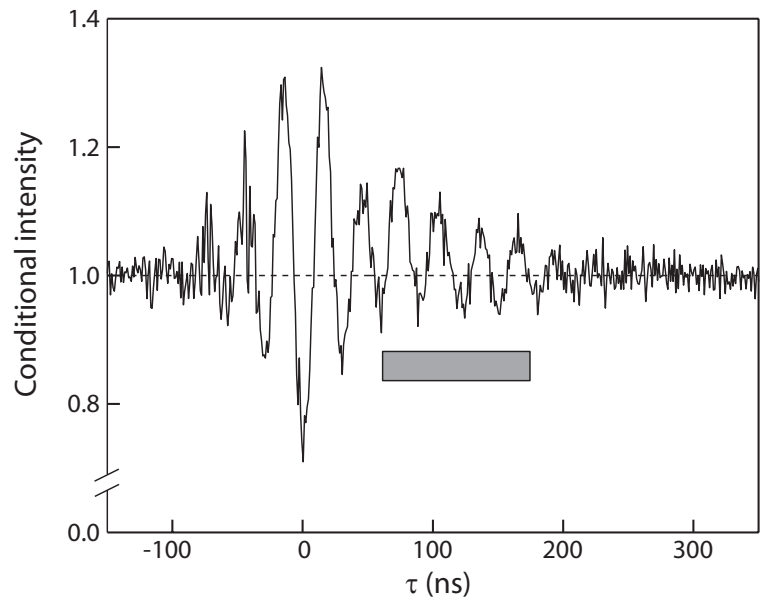

FIG. 10: Measured intensity correlation function. $(\Omega, g, \kappa) / 2 \pi=(34,5.1,3.7)$ MHz. The grey box indicates the application time of the square feedback pulse, $\Delta I=+3 \%, \tau_{\mathrm{fb}}=56 \mathrm{~ns}$, the intensity step was $120 \mathrm{~ns}$ long. The oscillation back to the steady state has been extended. Data binned into $1.0 \mathrm{~ns}$ bins.

indicates where the feedback pulse begins and ends. At the beginning of the feedback the system resides in the new steady state, the peak - present at the corresponding negative time - is suppressed. The effect of the $7 \mathrm{~ns}$ fall time on the return step of the pulse is to somewhat smoothly relax the system into the steady state [31], as is seen by the diminished size of the return oscillation at the end of the gray box.

One interesting question is how long we need to take data - meaning how many events do we need - before we know that the feedback has altered the oscillation. This is a question of signal to noise ratio. A typical oscillation that we suppress is about $10 \%$ larger than the steady 


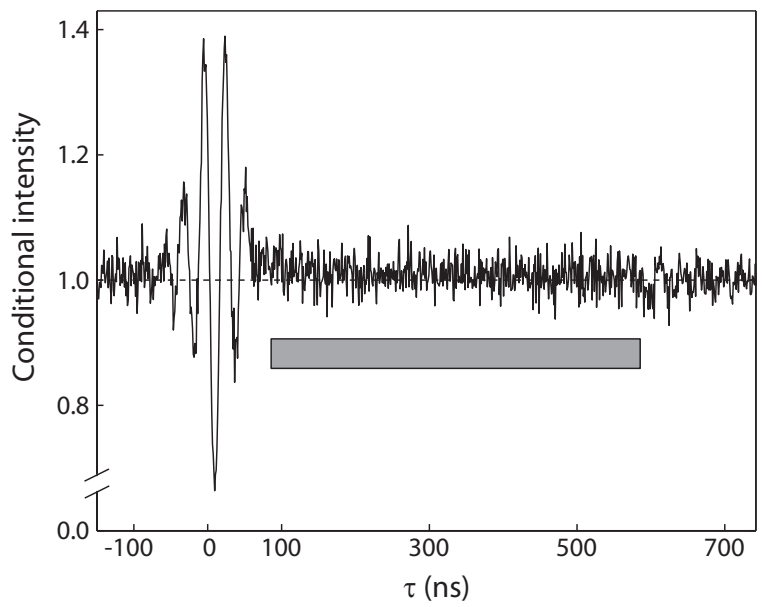

FIG. 11: Measured intensity correlation function, $(\Omega, g, \kappa) / 2 \pi=(37,6.1,4.9) \mathrm{MHz} . \quad$ A small intensity change, $\Delta I=+0.5 \%$, freezes the system into a steady state only slightly higher than the original. The dashed line marks the original steady state level at 1.0. The grey box indicates the application time of the square feedback pulse, $\tau_{\mathrm{fb}}=43 \mathrm{~ns}$, data binned into $2.0 \mathrm{~ns}$ bins.

state. This means that we need the uncertainty in the number of counts in that bin to be less than $10 \%$ (i.e. $\left.\sqrt{N_{\text {cts }}} / N_{\text {cts }}<0.10\right)$ before we can know if we have altered the number of counts in that bin. This occurs when $N_{\text {cts }}=100$. A $3 \sigma$ confidence level requires $N_{\text {cts }}=$ 900. If $\tau_{\mathrm{fb}} \approx 50 \mathrm{~ns}$, we shall take data at least in the region $\tau=0$ to $100 \mathrm{~ns}$, which is divided into 200 bins, so we need a total of 20000 events at the $1 \sigma$ level, where an event is defined as a start photon and a stop photon. We would also like to find out how long we have to take data until we know if the oscillation has been suppressed to, say $20 \%$ of its original level. Again, assuming an oscillation $10 \%$ larger than the background, we require $\sqrt{N_{\text {cts }}} / N_{\text {cts }}<0.02$. This occurs when $N_{\text {cts }}=2500$ for that bin, or after 500000 events.

Figure 12 shows the evolution of the $\tau=51 \mathrm{~ns}$ peak in Fig. 8 and its uncertainty as data accumulates. In this case the final value of the peak is $18 \%$ higher than the steady state. $1 \sigma$ confidence occurs at $N_{\text {cts }}=31$ and $3 \sigma$ at $N_{\mathrm{cts}}=278$.

\section{B. Step size and time-delay sensitivity study}

The size of the feedback intensity step must be correct to ensure that the system evolution is frozen. Fig. 13 shows the response of the system to different driving step sizes when the system is held in a steady state below the original drive. We are following the response of the first extreme of $g^{(2)}(\tau)$ after the application of a feedback pulse. Positive steps correspond to an increase in intensity, negative ones to a decrease.

The prediction (dashed line in Fig. 13) uses the dynamical equations conditioned on a cavity photoemission as in

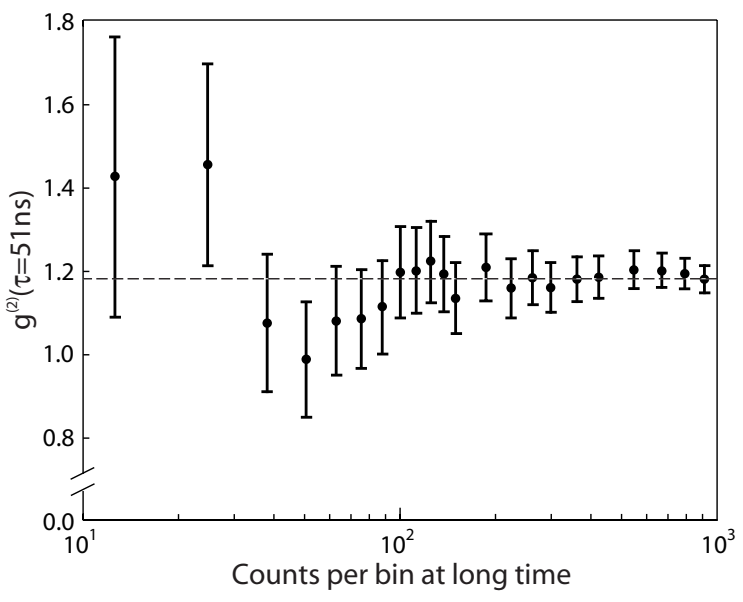

FIG. 12: Convergence of the $\tau=51$ ns peak in Fig. 8 with increasing sample size. The value of $g^{(2)}(\tau=51 \mathrm{~ns})$ is plotted in a logarithmic scale against the average number of counts per bin at long time. Error bars are $1 \sigma$. The horizontal line passes through the final measured value of $g^{(2)}(\tau=51 \mathrm{~ns})$.

Fig. 1, where now the system drive contains a step with a rise time. The rates in the model are the experimentally determined values $\Omega / 2 \pi=37 \mathrm{MHz}, \kappa / 2 \pi=3.7 \mathrm{MHz}$, and all broadening effects are incorporated by modifying the atomic polarization decay rate, $\gamma / 2 \pi=9.1 \mathrm{MHz}$. The amplitude is the deviation of the oscillation from the steady state value at the location of the peak identified for suppression. The steady state value is that corresponding to the system drive at the peak time. At any step amplitude other than the optimal one, the exchange of energy continues. For the system parameters of Fig. 13 the optimal suppression occurs at $\Delta I=-4.6 \%$. Notice that at $\Delta I=+4.6 \%$, the size of the oscillation is larger than that with no feedback at all; the feedback step augments the natural evolution of the system, leading to oscillation enhancement. The theory (dashed line) incorporates the measured shape of the pulse (at the point $-4.6 \%$ ), all sources of dephasing present in the system are modelled by the polarization decay rate $\gamma^{\prime} / 2 \pi=9.1$ $\mathrm{MHz}$. We adjust the number of atoms in the theory to a the point where the Rabi frequency $\Omega / 2 \pi$ agrees with the measurement. The plot shows both enhancement and suppression with quantitative agreement.

The feedback pulse must arrive at the correct time to ensure that the oscillation is properly suppressed. Fig. 14 shows the response of the system to feedback at various times, where $\tau_{\mathrm{fb}}=0$ is the time when the feedback protocol best suppresses the peak. The no feedback size of the peak was 0.08 above the steady state.

The rates in the model are the experimentally determined values $\Omega / 2 \pi=37 \mathrm{MHz}$, with $g / 2 \pi=5.1 \mathrm{MHz}$, $\kappa / 2 \pi=3.7 \mathrm{MHz}$. Notice that incorrectly timed feedback can enhance or suppress an oscillation, and the response of the system to the feedback timing is periodic, with period $2 \pi / \Omega \approx 27 \mathrm{~ns}$ in this case. At $\tau_{\mathrm{fb}}=0$ the system response - suppression - is analogous to the intensity 


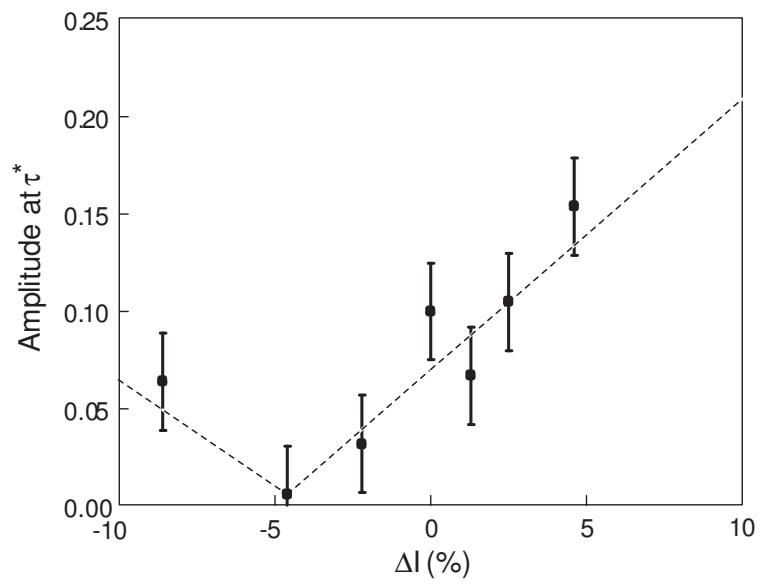

FIG. 13: Response of the system to feedback intensity steps of various sizes. $\tau^{\star}=70 \mathrm{~ns},(\Omega, g, \kappa) / 2 \pi=(37,5.1,3.7)$. For this set, the optimal suppression occurs at $\Delta I=-4.6 \%$. The dashed line is a theoretical prediction which includes the rise time of intensity step.

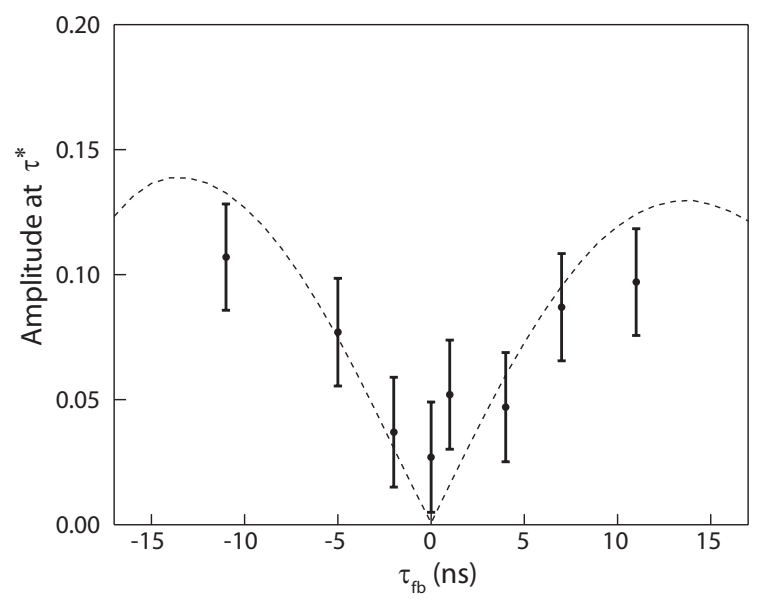

FIG. 14: Response of the system to feedback intensity steps at different times. $\tau_{\mathrm{fb}}=0$ is the optimal feedback time. The no feedback amplitude $(\Delta I=0)$ was 0.08 . The dashed line is the quantum theoretical prediction.

correlation displayed in Fig. 9, while at $\tau_{\mathrm{fb}} \approx \pm 12 \mathrm{~ns}$ the system displays enhancement, which is what happens in the intensity correlation of Fig. 10.

\section{Discussion}

An intensity step of any given length would allow us to produce an intensity correlation function with a suppressed region of that length of time. Photoemissions during the suspended time will be uncorrelated with $\tau=0$ and will arrive at the steady state rate determined by the updated drive. Subsequently returning the drive to the original value will result in an oscillation of the appropriate sign and amplitude.
It should be remembered that the intensity correlation measurement is constructed over many realizations of the system. The data set of Fig. 9, for example, contains about 5.4 million start counts, and 1.4 million coincidences in a $1 \mu \mathrm{s}$ window. This is necessary because of the rarity of detection events following the first detection. However, the feedback works to stabilize the state in every run of the experiment, as our ensemble average results verify. In this respect it is no different from classical feedback.

\section{CONCLUSION}

In this paper we have given a detailed treatment of the theory and experiment of feedback in the deep quantum regime of cavity QED. That is, the design of the feedback algorithm requires an understanding of the conditional state of the quantum system. It cannot be understood in terms of a semiclassical model with fluctuations, because the conditioning event, the detection of a photon emitted by the system, causes a change in the cavity field which is comparable to its mean. This is due to the fact that the driving in our system is very weak.

In our experiment, the nonequilibrium conditioned state following detection of a photon from the cavity 'rings down' to equilibrium, but at certain times (after the detection) the conditioned state can be frozen by the application of a feedback pulse. We showed that the pulse can be applied for an essentially arbitrary time, and when it ceases the system resumes its damped oscillations towards equilibrium with the same amplitude and phase that it had before it was frozen. We explored the sensitivity of the system to the time delay of the feedback step and to the amplitude of the feedback step, and found agreement with the theoretical model.

As we have emphasized in this paper, this feedback algorithm works only because at equilibrium our system is in a pure, entangled state. It is entangled because the atoms in our cavity constitute an anharmonic oscillator. If one were to try the experiment with two coupled harmonic oscillators, then there would be no entanglement as the steady state would be a joint coherent state. But then a photodetection would have no effect: there would be no nonequilibrium conditioned state to freeze. If one were to introduce noise so as to induce a correlated (but not entangled) mixed state, then a nonequilibrium conditioned state would arise. However, as we have shown in detail, the feedback could not work because a mixed state has more coefficients to stabilize than there are free parameters in our feedback.

The entanglement in the pure state of our system in equilibrium means that when a photon is detected, not only does the mean field of the cavity change (a quantum jump), but the mean polarization of our atoms also changes. These initial conditions for the after-jump conditioned state lead to specific predictions for the times at which the feedback pulse can be applied, which differ 
from the times which would apply if there were no entanglement and hence no jump in the atomic state. However; for the parameters of the current experiment, this time difference is too small to be reliably resolved.

Another direction for future work would be to consider feedback in a system that is driven off-resonance. When the cavity QED system is driven off-resonance the cavity field regresses to a steady state that is a combination of both quadratures of the electromagnetic field [46]. In order to apply feedback on this system one would have to not only change the intensity in the drive but also the system detuning in order to freeze the system into a new steady state. This might be accomplished while holding atoms in the cavity with an optical lattice and using the AC stark shift to vary the detuning of the atoms [47].

\section{Acknowledgments}

We would like to acknowledge the interest shown and help provided by S. Kuhr and C. Pancake. This work was supported by the NSF, NIST, the Australian Research Council and the State of Queensland.

\section{APPENDIX A: WHY THE STEADY STATE IS PURE}

Consider a system of damped and coupled oscillators (not necessarily harmonic) with ground state $|0\rangle$. We will take the coupling rates and damping rates to be of order unity. Now add weak linear driving of strength $\lambda \ll 1$. In this appendix we show that the steady state of the system $\rho_{\mathrm{ss}}$ is pure to order $\lambda^{2}$. That is, one can use the approximation

$$
\left|\psi_{\mathrm{ss}}\right\rangle=|0\rangle+\lambda\left|\psi_{1}\right\rangle+\lambda^{2}\left|\psi_{2}\right\rangle,
$$

where $\left|\psi_{1}\right\rangle$ and $\left|\psi_{2}\right\rangle$ are states (with norm of order unity) with one and two excitations respectively.

Consider unraveling the master equation of the system by quantum jumps (corresponding to the emission of a photon from the system). It is simple to verify that the no-jump evolution will take the system into a pure state, and that to second order in $\lambda$ it is of the form of Eq. (A1). Thus if we can show that the effect of the jumps is to add terms to $\rho_{\mathrm{ss}}$ of order $\lambda^{3}$ and higher, then we have established the desired results. That the extra terms from the jumps are of order $\lambda^{3}$ can be seen as follows.

First, the rate of jumps is of order $\lambda^{2}$ (this being the probability of excitation of the system times the damping rate which is of order unity). That is to say, jumps are rare events.

Second, the effect of a jump will be to once more create a state of the form $|0\rangle+O(\lambda)$. Thus, the after-jump state is different from $\left|\psi_{\text {ss }}\right\rangle$ only by an amount of order $\lambda$ at most.
Third, after a jump, the system will relax back to $\left|\psi_{\text {ss }}\right\rangle$ at a rate of order unity. That is to say, the nonequilibrium state will persist only for a time of order unity.

Putting these together, we see that excursions from $\left|\psi_{\mathrm{ss}}\right\rangle$ are only of order $\lambda$, and that the proportion of time the system spends making excursions is only of order $\lambda^{2}$. Thus the contribution of jumps to $\rho_{\mathrm{ss}}$ is of higher order than that from the no-jump stationary state (simplepure), as desired.

Note that if any of these three conditions are not satisfied, then this argument does not go through. In particular we will see in the following appendix that the jumps arising from phase noise cannot satisfy both conditions one and two.

\section{APPENDIX B: DYNAMICS OF NOISY HARMONIC OSCILLATORS}

In this appendix we derive Eq. (2.51). For the semiclassical system (two damped harmonic oscillators, one driven and the other experiencing phase noise) the master equation is

$$
\begin{aligned}
\dot{\rho}(t)= & \mathcal{E}\left[\hat{a}^{\dagger}-\hat{a}, \rho(t)\right]+g \sqrt{N}\left[\hat{a}^{\dagger} \hat{b}-\hat{a} \hat{b}^{\dagger}, \rho(t)\right] \\
& +2 \kappa \mathcal{D}[\hat{a}] \rho(t)+\gamma \mathcal{D}[\hat{b}] \rho(t)+\Gamma \mathcal{D}\left[\hat{b}^{\dagger} \hat{b}\right] \rho(t),
\end{aligned}
$$

where $\mathcal{D}[\hat{A}] \rho$ is defined in Eq. (2.6). The direct detection unraveling of this equation gives

$$
\rho_{1}(t+d t)=\frac{2 \kappa \hat{a} \rho(t) \hat{a}^{\dagger}}{\operatorname{Tr}\left[\hat{a}^{\dagger} \hat{a} \rho(t)\right]},
$$

for a detection, and the no-detection conditional state evolution, in the weak driving limit, is given by

$$
\begin{aligned}
\dot{\rho}_{0}(t)= & \mathcal{E}\left[\hat{a}^{\dagger}-\hat{a}, \rho_{0}(t)\right]+g \sqrt{N}\left[\hat{a}^{\dagger} \hat{b}-\hat{a} \hat{b}^{\dagger}, \rho_{0}(t)\right] \\
& -\kappa\left\{\hat{a}^{\dagger} \hat{a}, \rho_{0}(t)\right\}-\gamma\left\{\hat{b}^{\dagger} \hat{b}, \rho_{0}(t)\right\} / 2 \\
& +\Gamma \mathcal{D}\left[\hat{b}^{\dagger} \hat{b}\right] \rho_{0}(t) .
\end{aligned}
$$

where $\{\hat{A}, \hat{B}\}$ denotes the anti-commutator. Here we have used the fact that both terms $\hat{a} \rho_{0}(t) \hat{a}^{\dagger}$ and $\hat{b} \rho_{0}(t) \hat{b}^{\dagger}$ to dominant order in $\lambda$ have a negligible effect (see Appendix A). However, the phase jump term, $\hat{b}^{\dagger} \hat{b} \rho_{0}(t) \hat{b}^{\dagger} \hat{b}$, cannot be ignored. That is because the after-jump state will not be close to the ground state $|0\rangle$, but rather is guaranteed to contain at least one excitation of the $b$ mode. Thus the second condition of Appendix A is violated, and the steady state will not be pure. If one were to rewrite the phase diffusion term as $\mathcal{D}\left[\hat{b} \hat{b}^{\dagger}\right]$, and unravel accordingly, then the after-jump state would still be $|0\rangle+O(\lambda)$. However, the rate of jumps would then be of order unity, not of order $\lambda^{2}$, so that the first condition in Appendix A would be violated. 
To find the no-jump evolution we assume that, in the weak driving limit, we can truncate the Fock basis at 2 total excitations. That is,

$$
\rho_{0}(t)=\sum_{n, m, p, q}^{2} \lambda^{m+n+p+q} c_{m, p, n, q}(t)|m, p\rangle\langle n, q|,
$$

where $c_{n, m, p, q}(t)=c_{p, q, n, m}^{*}(t)$ are defined as follows: $c_{0,0,0,0}(t)=1, c_{0,0,0,1}(t)=f_{1}(t), c_{0,0,1,0}(t)=f_{2}(t)$, $c_{0,0,0,2}(t)=s_{1}(t), c_{0,0,1,1}(t)=s_{2}(t), c_{0,0,2,0}(t)=s_{3}(t)$, $c_{0,1,0,1}(t)=s_{4}(t), c_{0,1,1,0}(t)=s_{5}(t), c_{1,0,1,0}(t)=s_{6}(t)$, $c_{0,1,0,2}(t)=t_{1}(t), c_{0,1,1,1}(t)=t_{2}(t), c_{0,1,2,0}(t)=t_{3}(t)$, $c_{0,2,1,0}(t)=t_{4}(t), c_{1,0,1,1}(t)=t_{5}(t), c_{1,0,2,0}(t)=t_{6}(t)$, $c_{0,2,0,2}(t)=p_{1}(t), c_{0,2,1,1}(t)=p_{2}(t), c_{0,2,2,0}(t)=p_{3}(t)$, $c_{1,1,1,1}(t)=p_{4}(t), c_{1,1,2,0}(t)=p_{5}(t), c_{2,0,2,0}(t)=p_{6}(t)$, and for $m+p>2$ or $n+q>2, c_{m, p, n, q}=0$.

Substituting Eq. (B4) into Eq. (B3) gives the following set of differential equations

$$
\begin{aligned}
d_{t} f_{1}(t)= & -(\gamma+\Gamma) / 2 f_{1}(t)-g \sqrt{N} f_{2}(t) \\
d_{t} f_{2}(t)= & -\kappa f_{2}(t)+g \sqrt{N} f_{1}(t)+\mathcal{E} / \lambda \\
d_{t} s_{1}(t)= & -(\gamma+2 \Gamma) s_{1}(t)-\sqrt{2} g \sqrt{N} s_{2}(t), \\
d_{t} s_{2}(t)= & -(\kappa+\gamma / 2+\Gamma / 2) s_{2}(t)+\sqrt{2} g \sqrt{N}\left[s_{1}(t)\right. \\
& \left.-s_{3}(t)\right]+(\mathcal{E} / \lambda) f_{1}(t) \\
d_{t} s_{3}(t)= & -2 \kappa s_{3}(t)+\sqrt{2} g \sqrt{N} s_{2}(t) \\
& +\sqrt{2}(\mathcal{E} / \lambda) f_{2}(t) \\
d_{t} s_{4}(t)= & -\gamma s_{4}(t)-g \sqrt{N}\left[s_{5}(t)+s_{5}^{*}(t)\right] \\
d_{t} s_{5}(t)= & -(\kappa-\gamma / 2-\Gamma / 2) s_{5}(t)+g \sqrt{N}\left[s_{4}(t)\right. \\
& \left.-s_{6}(t)\right]+(\mathcal{E} / \lambda) f_{1}^{*}(t) \\
d_{t} s_{6}(t)= & -2 \kappa s_{6}(t)+g \sqrt{N}\left[s_{5}(t)+s_{5}^{*}(t)\right] \\
& +(\mathcal{E} / \lambda)\left[f_{2}(t)+f_{2}^{*}(t)\right]
\end{aligned}
$$

$$
\begin{aligned}
& d_{t} t_{1}(t)=-(3 \gamma / 2+\Gamma / 2) t_{1}(t)-g \sqrt{N}\left[t_{4}^{*}(t)\right. \\
& \left.+\sqrt{2} t_{2}(t)\right] \\
& d_{t} t_{2}(t)=-(\kappa+\gamma) t_{2}(t)+g \sqrt{N}\left[\sqrt{2} t_{1}(t)-t_{5}(t)\right. \\
& \left.-\sqrt{2} t_{3}(t)\right]+(\mathcal{E} / \lambda) s_{4}(t) \\
& d_{t} t_{3}(t)=-(2 \kappa+\gamma / 2+\Gamma / 2) t_{3}(t)+g \sqrt{N}\left[\sqrt{2} t_{2}(t)\right. \\
& \left.-t_{6}(t)\right]+\sqrt{2}(\mathcal{E} / \lambda) s_{5}(t), \\
& d_{t} t_{4}(t)=-(\kappa+\gamma+2 \Gamma) t_{4}(t)+g \sqrt{N}\left[t_{1}^{*}(t)\right. \\
& \left.-\sqrt{2} t_{5}^{*}(t)\right]+\mathcal{E} s_{1}^{*}(t) \\
& d_{t} t_{5}(t)=-(2 \kappa-\gamma / 2-\Gamma / 2) t_{5}(t)+g \sqrt{N}\left[t_{2}(t)\right. \\
& \left.-\sqrt{2} t_{6}(t)+\sqrt{2} t_{4}^{*}(t)\right]+(\mathcal{E} / \lambda)\left[s_{2}(t)\right. \\
& \left.+s_{5}^{*}(t)\right] \\
& d_{t} t_{6}(t)=-3 \kappa t_{6}(t)+g \sqrt{N}\left[t_{3}(t)+\sqrt{2} t_{5}(t)\right] \\
& +\mathcal{E}\left[s_{3}(t)+\sqrt{2} s_{6}(t)\right], \\
& d_{t} p_{1}(t)=-2 \gamma p_{1}(t)-\sqrt{2} g \sqrt{N}\left[p_{2}^{*}(t)+p_{2}(t)\right] \text {, } \\
& d_{t} p_{2}(t)=-(\kappa+3 \gamma / 2+\Gamma / 2) p_{2}(t)+\sqrt{2} g \sqrt{N}\left[p_{1}(t)\right. \\
& \left.-p_{3}(t)-p_{4}(t)\right]+(\mathcal{E} / \lambda) t_{1}^{*}(t), \\
& d_{t} p_{3}(t)=-(2 \kappa+\gamma+-2 \Gamma) p_{3}(t)+\sqrt{2} g \sqrt{N}\left[p_{2}(t)\right. \\
& \left.-p_{5}(t)\right]+\sqrt{2}(\mathcal{E} / \lambda) t_{4}(t), \\
& d_{t} p_{4}(t)=-(2 \kappa+\gamma) p_{4}(t)+\sqrt{2} g \sqrt{N}\left[p_{2}(t)+p_{2}^{*}(t)\right. \\
& \left.-p_{5}(t)-p_{5}^{*}(t)\right]+(\mathcal{E} / \lambda)\left[t_{2}(t)+t_{2}^{*}(t)\right],(\mathrm{B} 22) \\
& d_{t} p_{5}(t)=-(3 \kappa+\gamma / 2+\Gamma / 2) p_{5}(t)+\sqrt{2} g \sqrt{N}\left[p_{3}(t)\right. \\
& \left.-p_{6}(t)+p_{4}(t)\right]+(\mathcal{E} / \lambda)\left[t_{2}^{*}+\sqrt{2} t_{5}^{*}\right] \text {, } \\
& d_{t} p_{6}(t)=-4 \kappa p_{6}(t)+\sqrt{2} g \sqrt{N}\left[p_{5}(t)+p_{5}^{*}(t)\right] \\
& +\sqrt{2}(\mathcal{E} / \lambda)\left[t_{6}(t)+t_{6}^{*}(t)\right]
\end{aligned}
$$

which when solved gives the weak driving solution to the no-jump evolution. The steady state is found by setting all the derivatives to zero. We do not present this as the expression for the steady state is rather lengthy.

The conditional state representing a detection at time $t=0$ (given that the system was originally in its steady state) to order $\lambda^{2}$ is

$$
\begin{aligned}
\rho_{c}(0)= & \frac{\hat{a} \rho_{\mathrm{ss}} \hat{a}^{\dagger}}{\operatorname{Tr}\left[\hat{a}^{\dagger} \hat{a} \rho_{\mathrm{ss}}\right]} \\
= & |0,0\rangle\langle 0,0|+\frac{\lambda^{2}}{s_{6}^{\mathrm{ss}}}\left[\sqrt{2} p_{5}^{\mathrm{ss}}|0,1\rangle\langle 1,0|+\text { H.c }\right] \\
& +\frac{\lambda}{s_{6}^{\mathrm{ss}}}\left[t_{5}^{\mathrm{ss}}|0,0\rangle\left\langle 0,1\left|+\sqrt{2} t_{6}^{\mathrm{ss}}\right| 0,0\right\rangle\langle 1,0|+\text { H.c }\right] \\
& +\frac{\lambda^{2}}{s_{6}^{\mathrm{ss}}}\left[p_{4}^{\mathrm{ss}}|0,1\rangle\left\langle 0,1\left|+2 p_{6}^{\mathrm{ss}}\right| 1,0\right\rangle\langle 1,0|\right]
\end{aligned}
$$

where $s_{6}^{\text {ss }}$, for example, represents the steady state value of the coherence $s_{6}(\tau)$. Thus the conditional state at time $\tau$ is given by Eq. (2.51) with the following initial 
conditions

$$
\begin{aligned}
& f_{1}(0)=t_{5}^{\mathrm{ss}} / s_{6}^{\mathrm{ss}}, \\
& f_{2}(0)=\sqrt{2} t_{6}^{\mathrm{ss}} / s_{6}^{\mathrm{ss}}, \\
& s_{4}(0)=p_{4}^{\mathrm{ss}} / s_{6}^{\mathrm{ss}}, \\
& s_{5}(0)=\sqrt{2} p_{5}^{\mathrm{ss}} / s_{6}^{\mathrm{ss}}, \\
& s_{6}(0)=2 p_{6}^{\mathrm{ss}} / s_{6}^{\mathrm{ss}} .
\end{aligned}
$$

[1] Y. Yamamoto, N. Imoto, and S. Machida, Phys. Rev. A 33, 3243 (1986).

[2] H. A. Haus and Y. Yamamoto, Phys. Rev. A 34, 270 (1986).

[3] J. H. Shapiro, G. Saplakoglu, S. T. Ho, P. Kumar, B. E. A. Saleh, and M. C. Teich, J. Opt. Soc. Am. B 4, 1604 (1987).

[4] H. M. Wiseman and G. J. Milburn, Phys. Rev. Lett. 70, 548 (1993).

[5] H. M. Wiseman, Phys. Rev. A 49, 2133 (1994).

[6] Cavity Quantum Electrodynamics, Advances in Atomic, Molecular, and Optical Physics, edited by P. R. Berman (Academic Press, Boston, 1994), supplement 2.

[7] H. J. Carmichael, R. J. Brecha, and P. R. Rice, Opt. Commun. , 82, 73 (1991).

[8] J. E. Reiner, W. P. Smith, L. A. Orozco, H. J. Carmichael, and P. R. Rice, J. Opt. Soc. Am. B 18, 1911 (2001).

[9] W. P. Smith, J. E. Reiner, L. A. Orozco, S. Kuhr, and H. M. Wiseman, Phys. Rev. Lett. 89, 133601 (2002).

[10] H. J. Carmichael, An Open Systems Approach to Quantum Optics, Lecture Notes in Physics (Springer-Verlag, Berlin, 1993), Vol. 18.

[11] J. J. Slosser and G. J. Milburn, Phys. Rev. Lett. 75, 418 (1995).

[12] P. Tombesi and D. Vitali, Phys. Rev. A 51, 4913 (1995).

[13] P. Goetsch, P. Tombesi, and D. Vitali, Phys. Rev. A 54, 4519 (1996).

[14] D. B. Horoshko and S. Y. Kilin, Phys. Rev. Lett. 78, 840 (1997).

[15] J. Wang and H. M. Wiseman, Phys. Rev. A 64, 063810 (2001).

[16] S. Zippilli, D. Vitali, P. Tombesi, and J. M. Raimond, Phys. Rev. A 67, 052101 (2003).

[17] J. A. Dunningham, H. M. Wiseman, and D. F. Walls, Phys. Rev. A 55, 1398 (1997).

[18] S. Mancini and P. Tombesi, Phys. Rev. A 56, 2466 (1997).

[19] S. Mancini, D. Vitali, and P. Tombesi, Phys. Rev. Lett. 80, 688 (1998).

[20] L. K. Thomsen, S. Mancini, and H. M. Wiseman, Phys. Rev. A 65, 061801 (2002).

[21] J. E. Reiner, H. M. Wiseman, and H. Mabuchi, Phys. Rev. A 67, 042106 (2003).

[22] H. M. Wiseman, Phys. Rev. Lett. 75, 4587 (1995).

[23] A. C. Doherty and K. Jacobs, Phys. Rev. A 60, 2700 (1999).

[24] A. C. Doherty, S. Habib, K. Jacobs, H. Mabuchi, and
S. M. Tan, Phys. Rev. A 62, 012105 (2000).

[25] H. M. Wiseman, S. Mancini, and J. Wang, Phys. Rev. A 66, 013807 (2002).

[26] M. A. Armen, J. K. Au, J. K. Stockton, A. C. Doherty, and H. Mabuchi, Phys. Rev. Lett. 89, 133602 (2002).

[27] H. J. Carmichael, H. M. Castro-Beltran, G. T. Foster, and L. A. Orozco, Phys. Rev. Lett. 85, 1855 (2000).

[28] G. T. Foster, S. L. Mielke, and L. A. Orozco, Phys. Rev. A 61, 053821 (2000).

[29] G. T. Foster, W. P. Smith, J. E. Reiner, and L. A. Orozco, Phys. Rev. A 66, 033807 (2002).

[30] H. M. Wiseman and G. J. Milburn, Phys. Rev. A 47, 1652 (1993).

[31] R. J. Brecha, L. A. Orozco, M. G. Raizen, M. Xiao, and H. J. Kimble, J. Opt. Soc. Am. B 12, 2329 (1995).

[32] S. L. Mielke, G. T. Foster, J. Gripp, and L. A. Orozco, Opt. Lett. 22, 325 (1997).

[33] C. J. Hood, R. W. Lynn, A. C. Doherty, A. S. Parkins, and H. J. Kimble, Science 287, 1447 (2000).

[34] P. W. H. Pinkse, T. Fischer, P. Maunz, and G. Rempe, Nature 404, 365 (2000).

[35] G. R. Guthohrlein, M. Keller, K. Hayasaka, W. Lange, and H. Walther, Nature 414, 49 (2001).

[36] A. B. Mundt, A. Kreuter, C. Becher, D. Leibfried, J. Eschner, F. Schmidt-Kaler, and R. Blatt, Phys. Rev. Lett. 89, 103001 (2002).

[37] H. J. Carmichael and B. C. Sanders, Phys. Rev. A 60, 2497 (1999).

[38] G. Rempe, R. J. Thompson, R. J. Brecha, W. D. Lee, and H. J. Kimble, Phys. Rev. Lett. 67, 1727 (1991).

[39] J. E. Reiner, F. M. Dimler, and L. A. Orozco, J. of Optics B: Quantum Semiclass. Opt. 6, 135 (2004).

[40] J. P. Clemens and P. R. Rice, Phys. Rev. A 61, 063810 (2000).

[41] G. T. Foster, S. L. Mielke, and L. A. Orozco, J. Opt. Soc. Am. B 15, 2646 (1998).

[42] F. Davidson and L. Mandel, J. App. Phys. 39, 62 (1968).

[43] H. J. Carmichael, S. Singh, R. Vyas, and P. R. Rice, Phys. Rev. A 39, 1200 (1989).

[44] A. L. Lacaita, F. Zappa, S. Bigliardi, and M. Manfredi, IEEE Trans. Electron Devices ED40, 577 (1993).

[45] T. B. Pittman, B. C. Jacobs, and J. D. Franson, Phys. Rev. A 66, 052305 (2002).

[46] W. P. Smith and L. A. Orozco, J. of Optics B: Quantum Semiclass. Opt. 6, 127 (2004).

[47] M. L. Terraciano, W. P. Smith, and L. A. Orozco, CLEOQELS 2003 Conference Program QTuG6, 122 (2003). 